\title{
Uniformly local spaces and refinements of the classical Sobolev embedding theorems
}

\author{
Patrick J. Rabier
}

\begin{abstract}
We prove that if $f$ is a distribution on $\mathbb{R}^{N}$ with $N>1$ and if $\partial_{j} f \in L^{p_{j}, \sigma_{j}} \cap L_{u l o c}^{N, 1}$ with $1 \leq p_{j} \leq N$ and $\sigma_{j}=1$ when $p_{j}=1$ or $N$, then $f$ is bounded, continuous and has a finite constant radial limit at infinity. Here, $L^{p, \sigma}$ is the classical Lorentz space and $L_{\text {uloc }}^{p, \sigma}$ is a "uniformly local" subspace of $L_{l o c}^{p, \sigma}$ larger than $L^{p, \sigma}$ when $p<\infty$.

We also show that $f \in \mathcal{B U C}$ if, in addition, $\partial_{j} f \in L^{p_{j}, \sigma_{j}} \cap L_{u l o c}^{q}$ with $q>N$ whenever $p_{j}<N$ and that, if so, the limit of $f$ at infinity is uniform if the $p_{j}$ are suitably distributed. Only a few special cases have been considered in the literature, under much more restrictive assumptions that do not involve uniformly local spaces $\left(p_{j}=N\right.$ and $f$ vanishing at infinity, or $\partial_{j} f \in L^{p} \cap L^{q}$ with $p<N<q$ ).

Various similar results hold under integrability conditions on the higher order derivatives of $f$. All of them are applicable to $g * f$ with $g \in L^{1}$ and $f$ as above, or under weaker assumptions on $f$ and stronger ones on $g$. When $g$ is a Bessel kernel, the results are provably optimal in some cases.
\end{abstract}

\section{Introduction}

All the function spaces with unspecified domain are over $\mathbb{R}^{N}$ and differentiations are understood in the sense of distributions. If $f \in \mathcal{D}^{\prime}$ (distributions) and $\nabla f \in\left(L^{p}\right)^{N}$, then $f$ is uniformly continuous if $p>N$ and $f \in \mathcal{B U C}$ (bounded uniformly continuous functions) if $N=p=1$, but no restriction on $p$ implies that $f$ is bounded when $N>1$.

The general purpose of this paper is to discuss conditions on $\nabla f$ and, possibly, on the higher order derivatives of $f$ (but not on $f$ ) that imply $f \in \mathcal{C}_{b}$ (bounded continuous functions) or $f \in \mathcal{B U C}$ when $N>1$. These conditions also imply that $f$ lution.

Key words and phrases: Lebesgue point, uniformly local space, Sobolev embedding, convo2010 Mathematics Subject Classification: 46E35, $26 \mathrm{~B} 35$. 
has a finite constant limit at infinity, be that uniform or in a weaker radial sense. This yields new Sobolev embedding theorems in cases when the classical ones do not already ensure the continuity of $f$, for instance when $\nabla f \in\left(L^{p}\right)^{N}$ with $p \leq N$, but also in the anisotropic setting when the partial derivatives of $f$ belong to different Lorentz spaces.

Throughout the paper, we assume without loss of generality that

(1.1) the representatives of $L_{l o c}^{1}$ functions are always chosen so that they coincide with the limit of their Lebesgue averages at every Lebesgue point.

In particular, if every point of $f$ is a Lebesgue point, there is only one representative of $f$ complying with (1.1), which is identified with $f$. This will make it unnecessary to qualify properties "after modifying $f$ on a set of measure 0 ".

The main result - insofar as everything else depends on it - is part (i) of the following theorem, but part (ii) will be the starting point of the discussion. The notation $L^{N, 1}$ refers to the classical Lorentz space.

Theorem 1.1. (i) If $\nabla f \in\left(L_{l o c}^{1}\right)^{N}$ and $|x|^{1-N_{*}} *|\nabla f| \in L^{\infty}$, then: $(i-1)\left(|x|^{1-N} *|\nabla f|\right)(z) \leq\left\||x|^{1-N_{*}} *|\nabla f|\right\|_{\infty}$ for every $z \in \mathbb{R}^{N}$.

( $i$-2) $f \in W_{\text {loc }}^{1,1}$ and every $z \in \mathbb{R}^{N}$ is a Lebesgue point of $f$ (hence, by (1.1), $f$ is uniquely determined).

$\left(i\right.$-3) $f \in L^{\infty}$ and there is a constant $C>0$ independent of $f$ such that

$$
\sup |f|=\|f\|_{\infty} \leq \inf |f|+C\left\||x|^{1-N} *|\nabla f|\right\|_{\infty} .
$$

(ii) If $\nabla f \in\left(L^{N, 1}\right)^{N}$, then $f \in \mathcal{B U C}$ and there is a constant $C>0$ independent of $f$ such that $\|f\|_{\infty} \leq \inf |f|+C\|\nabla f\|_{N, 1}$.

As a result of (i-2), $f$ is approximately continuous, that is, continuous when $\mathbb{R}^{N}$ is equipped with the density topology (the open subsets are $\varnothing$ and the measurable subsets with density 1 at each point), but this will not be used later.

Part (ii) of Theorem 1.1 is essentially a special case of part (i), but not the only useful one. A related result by Cianchi and Pick [4, Theorem 3.5 (ii)] states that if $\Omega \subset \mathbb{R}^{N}$ is an open subset of finite measure satisfying a technical geometric condition and if $\nabla f \in\left(L^{N, 1}(\Omega)\right)^{N}$, then $f \in L^{\infty}(\Omega)$. However, $\Omega$ must actually be bounded, for otherwise $f(x)=\left(1+|x|^{2}\right)^{1 / 2}$, with gradient in $L^{\infty}(\Omega) \subset L^{N, 1}(\Omega)$ when $\Omega$ has finite measure, is a counter-example.

In turn, if $\Omega$ is bounded and functions with gradient in $L^{N, 1}(\Omega)$ have extensions to $\mathbb{R}^{N}$ with gradient in $L^{N, 1}$ (for instance if $\Omega$ has a Lipschitz boundary), then $f \in$ $\mathcal{C}(\bar{\Omega}) \subset L^{\infty}(\Omega)$ also follows from Stein's remark in [26] that functions with gradient in $L_{l o c}^{N, 1}$ are continuous (and a.e. differentiable). This does not prove that $f$ is 
bounded or uniformly continuous when $\Omega=\mathbb{R}^{N}$, although this follows from Tartar [28, Theorem 8 and Remark 9] under the additional assumption that $f$ "vanishes at infinity", i.e., $\left|\left\{x \in \mathbb{R}^{N}:|f(x)|>a\right\}\right|<\infty$ for every $a>0$ ([13]). By Theorem 1.1 (ii), this additional assumption is not needed.

If $1 \leq p<N<q \leq \infty$, then $L^{p} \cap L^{q} \subset L^{N, 1}$ and so, by Theorem 1.1 (ii), $f \in \mathcal{B U C}$ if $\nabla f \in\left(L^{p} \cap L^{q}\right)^{N}$. This is implicit in Galdi [9, p. 80], who shows that $f$ (continuous since $q>N$ ) has a uniform finite constant limit at infinity. As we shall see, this remains true when $N>1$ and $\nabla f \in\left(L^{N, 1}\right)^{N}$ (Theorem 3.7) and, in a weaker form, even when it is only assumed that $|x|^{1-N_{*}}|\nabla f| \in L^{\infty}$ (Theorem 3.1).

An interesting by-product (see Theorem 3.10 for a fuller statement) is that if $N>1$ and $\nabla^{N} f \in\left(L^{1}\right)^{N^{N}}$, then $f$ differs from a polynomial $P_{f}$ of degree at most $N-1$ by a $\mathcal{B U C}$ function tending uniformly to 0 at infinity. The proof shows how $P_{f}$ can be calculated. This is well-known, with $P_{f}=0$, when $f \in W^{N, 1}$ but trivially false in general form when $N=1$ (if $f^{\prime} \in L^{1}$, then $\lim _{|x| \rightarrow \infty} f(x)$ need not exist since $\lim _{x \rightarrow \pm \infty} f(x)$ need not be equal).

In Section 4, we turn our attention to the more general case when $\partial_{j} f \in L^{p_{j}, \sigma_{j}}$ with $1 \leq p_{j} \leq N$ and $\sigma_{j}=1$ when $p_{j}=1$ or $N$ (and $1 \leq j \leq N$, which is always implicit in the sequel). If so, $f$ need not be bounded or continuous, even if $\sigma_{j}=p_{j}=p<N$ and $f \in W^{1, p}$. Nevertheless, we prove the sharper form of Lusin's theorem that $f$ is continuous and bounded outside a set of arbitrarily small measure (Theorem 4.2 (i)) and, later, that $f$ is a finite sum of derivatives of bounded functions (Theorem 5.2 (i)).

A remark of greater consequence is that $|x|^{1-N} *|\nabla f| \in L^{\infty}$ (so that Theorem 1.1 (i) is applicable) if, in addition, $\partial_{j} f$ is locally in $L^{N, 1}$, albeit in a uniform way. To clarify this statement, define the uniformly local Lorentz space

$$
L_{u l o c}^{p, \sigma}:=\left\{h \in L_{l o c}^{p, \sigma}: \sup _{z \in \mathbb{R}^{N}}\|h\|_{p, \sigma, B_{1}(z)}<\infty\right\}
$$

where $B_{1}(z)$ is the unit ball with center $z$. When $\sigma=p$, the spaces $L_{u l o c}^{p}=L_{u l o c}^{p, p}$ were first introduced by Kato [12] for different purposes.

A generalization of Theorem 1.1 (ii) asserts that if $\partial_{j} f \in L^{p_{j}, \sigma_{j}} \cap L_{u l o c}^{N, 1}$ with $1 \leq p_{j} \leq N$ and $\sigma_{j}=1$ if $p_{j}=1$ or $N$, then $f \in \mathcal{C}_{b}$ and $f$ has a constant radial limit at infinity (Theorem 4.2 (ii)) and that $f \in \mathcal{B U C}$ if $\partial_{j} f \in L^{p_{j}, \sigma_{j}} \cap L_{u l o c}^{q}$ for some $q>N$ and every $j$ such that $p_{j}<N$ (Theorem 4.2 (iii)). Furthermore, the limit of $f$ at infinity is uniform if the $p_{j}$ are suitably distributed (Theorem 4.5). Both Theorem 1.1 (ii) and Theorem 3.7 are recovered when $p_{j}=N$. Anecdotally, the behavior at infinity of Beppo-Levi functions (functions $f$ with $\nabla^{k} f \in\left(L^{p}\right)^{N^{k}}$ for some $k \geq 1$ and variants) has been discussed since the 1950s (Deny and Lions [5], Uspenskii [30], Fefferman [8], Mizuta [16], Galdi [9], among others), but we are not aware of prior results for the anisotropic case. 
Inasmuch as the UL spaces are linearly ordered by inclusion, it is fairly easy to obtain generalizations when UL integrability conditions are now required of some higher order partial derivatives of $f$ instead of $\nabla f$ (Theorem 4.7). When these conditions are expressed in terms of the Lebesgue spaces, direct proofs bypassing UL spaces can only be given under very restrictive assumptions (Corollary 4.8 and subsequent comments).

Lastly, in Section 5, we investigate the boundedness and continuity of $g * f$ when $g \in L^{1}$ or better and $\partial_{j} f \in L^{p_{j}, \sigma_{j}}, 1 \leq p_{j} \leq N$ and $\sigma_{j}=1$ if $p_{j}=1$ or $N$, possibly without UL integrability conditions (Theorem 5.2, Theorem 5.6, Theorem 5.7). Since $f$ need not belong to any $L^{p, \sigma}$, it is not obvious that $g * f$ is defined as a distribution. This follows from results of Schwartz [24] and, rather unexpectedly, Theorem 1.1. Theorem 5.6 also depends upon a partial generalization of Young's theorem, to the effect that $g * h \in L_{u l o c}^{p, \sigma}$ if $g \in L^{1}$ and $h \in L_{u l o c}^{p, \sigma}$ (Lemma 5.5). No such generalization exists when $g \in L^{q}$ with $q>1$.

When $g=G_{\lambda}$ is a Bessel kernel (Example 5.3), the results can be corroborated by the standard elliptic theory if $\lambda=2 k$ with $k \in \mathbb{N}$ and $\sigma_{j}=p_{j}=p>1$ is independent of $j$ and, if so, they are provably optimal. To our best knowledge, they are new in the other cases: $p=1$, or $\sigma_{j}>p$ for some $j$, or $p_{j}$ distinct, or $\lambda \neq 2 k$.

The assumption $|x|^{1-N} *|\nabla f| \in L^{\infty}$ in Theorem 1.1 (i) may be viewed, albeit not literally, as the "exponent 0 " case of a condition introduced by the author in [22] to discuss Hölder continuity beyond Morrey's theorem. However, since the approach of [22] breaks down when the Hölder exponent vanishes and since new issues arise, key technicalities must be modified and other arguments must be developed.

Many results involve some Poincaré-Wirtinger inequality (classical, on the unit sphere, in Lorentz spaces, in UL spaces, in anisotropic spaces). Proofs are given, but stripped of the obvious recurrent details, when a convenient reference could not be found.

It is notorious that distributions with locally integrable gradient are themselves locally integrable ([15, p. 23]) and therefore functions in $W_{l o c}^{1,1}$. This will henceforth be used without further mention.

Throughout the paper, the notation is standard or defined as needed. The euclidean open ball with radius $r>0$ centered at $z \in \mathbb{R}^{N}$ is denoted $B_{r}(z)$ and abbreviated $B_{r}$ when $z=0$. The $L^{p}$ norm on the (Lebesgue) measurable set $E \subset \mathbb{R}^{N}$ is denoted $\|\cdot\|_{p, E}$ and abbreviated $\|\cdot\|_{p}$ when $E=\mathbb{R}^{N}$. Also, $p^{\prime}$ always refers to the Hölder conjugate of $p\left(1 / p^{\prime}=1-1 / p\right)$ and, if $1 \leq p<N$, we set $p^{*}:=N p /(N-p)$. Whether $|\cdot|$ refers to the euclidean norm or to the measure on $\mathbb{R}^{N}$ or $\mathbb{S}^{N-1}$ will always be obvious from the context.

Recall also that if $E \subset \mathbb{R}^{N}$ is measurable and $h \in L^{p, \sigma}(E)$, the quasi-norm $\|h\|_{p, \sigma, E}$, abbreviated $\|h\|_{p, \sigma}$ if $E=\mathbb{R}^{N}$, is defined by $\left(\int_{0}^{\infty}\left(t^{1 / p} h^{*}(t)\right)^{\sigma} d t / t\right)^{1 / \sigma}$ if 
$\sigma<\infty$ and by $\sup _{t>0} t^{1 / p} h^{*}(t)$ if $\sigma=\infty$, where $h^{*}$ is the decreasing rearrangement of $h$. The quasi-norm $\|\cdot\|_{p, \sigma, E}$ is a norm if $\sigma \leq p$. As is customary, if $F \in\left(L^{p, \sigma}(E)\right)^{N}$, we set $\|F\|_{p, \sigma, E}:=\||F|\|_{p, \sigma, E}$. For all the pairs $(p, \sigma)$ considered in this paper, $L^{p, \sigma}(E)$ is a Banach space for a norm equivalent to the quasi-norm $\|\cdot\|_{p, \sigma, E}$, but we shall not need its explicit definition. Further details can be found in [2], [3] and many other standard texts.

Up to a constant multiple, the convolution with $|x|^{\lambda-N}, 0<\lambda<N$ is usually referred to as the Riesz potential $I_{\lambda}$. Thus, the convolution with $|x|^{1-N}$ is essentially the Riesz potential $I_{1}$. This terminology will occasionally be used when making reference to the literature.

\section{Proof of Theorem 1.1}

The starting point is the obvious remark that a function is bounded if (and only if) its oscillation is bounded. The bulk of the proof consists in showing that (a) on any subset $S \subset \mathbb{R}^{N}$, the bounded oscillation property can be reduced to estimates for the averages $\left|B_{\rho}\right|^{-1} \int_{B_{\rho}(z)}|f(x)-f(z)| d x$ independent of $z \in S$ and of $\rho>0$ (Lemma 2.4) and that (b) these averages can be controlled, uniformly in $\rho$, by a constant multiple of $|x|^{1-N} *|\nabla f|$ (Theorem 2.3). These features will be needed in other proofs as well.

In what follows, $L_{|x|^{1-N}}^{1}$ denotes the weighted Lebesgue space

$$
L_{|x|^{1-N}}^{1}:=\left\{h \in L_{l o c}^{1}:|x|^{1-N} h \in L^{1}\right\},
$$

equipped with the natural norm $\|h\|_{1,|x|^{1-N}}:=\left\||x|^{1-N} h\right\|_{1}$. Since $|x|^{1-N}$ is an $A_{1}$ weight (as is $|x|^{\delta}$ when $-N<\delta \leq 0$; the definition is unimportant here), the following approximation lemma is a special case of Muckenhoupt and Wheeden [17, Lemma 8]. See also Turesson [29, Theorem 2.1.4].

Lemma 2.1. Suppose that $\eta \in \mathcal{C}_{0}^{\infty}$ is a nonnegative and nonincreasing function of $|x|$ and that $\int_{\mathbb{R}^{N}} \eta=1$. Set $\eta_{n}(x):=n^{N} \eta(n x)$. If $h \in L_{|x|^{1-N}}^{1}$, then $\eta_{n} * h \in L_{|x|^{1-N}}^{1}$ and $\eta_{n} * h \rightarrow h$ in $L_{|x|^{1-N}}^{1}$.

As is customary, we use the notation $f(r, \omega)$ for the expression of a function $f(x)$ in spherical coordinates, i.e., $f(r, \omega):=f(r \omega)$.

Lemma 2.2. If $f \in L_{l o c}^{1}$ and $\nabla f \in\left(L_{|x|^{1-N}}^{1}\right)^{N}$, there is a constant $c$ such that

$$
\|f(r, \cdot)-c\|_{1, \mathbb{S}^{N-1}} \leq\left\||x|^{1-N}|\nabla f|\right\|_{1, B_{r}},
$$

for every $r>0$. 
Proof. If $N=1$, then $L_{|x|^{1-N}}^{1}=L^{1}$, so that $f$ is absolutely continuous and (2.2) holds with $c=f(0)$. From now on, $N>1$.

Suppose first that $f \in \mathcal{C}^{\infty}$. Then, $f(r, \omega)=f(0)+\int_{0}^{r} \partial_{r} f(t, \omega) d t$, where $\partial_{r} f(x)=$ $\nabla f(x) \cdot|x|^{-1} x$ is the radial derivative of $f$. Since $\left|\partial_{r} f(x)\right| \leq|\nabla f(x)|$, it follows that $\|f(r, \cdot)-f(0)\|_{1, \mathbb{S}^{N-1}} \leq \int_{\mathbb{S}^{N-1}} d \omega \int_{0}^{r}|\nabla f(r, \omega)| d t=\left\||x|^{1-N}|\nabla f|\right\|_{1, B_{r}}$. This proves (2.2) with $c=f(0)$.

In general, note that $L_{|x|^{1-N}}^{1} \subset L_{l o c}^{1}$, so that $f \in W_{l o c}^{1,1}$. As a result, the restriction (trace) of $f$ is defined in $L^{1}\left(r \mathbb{S}^{N-1}\right)$ for every $r>0$. Let $\eta_{n}$ be the mollifying sequence of Lemma 2.1. Then, $f_{n}:=\eta_{n} * f \in \mathcal{C}^{\infty}$ tends to $f$ in $W_{l o c}^{1,1}$, so that $f_{n}$ tends to $f$ in $L^{1}\left(r \mathbb{S}^{N-1}\right)$. Equivalently, $\lim _{n \rightarrow \infty} f_{n}(r, \cdot)=f(r, \cdot)$ in $L^{1}\left(\mathbb{S}^{N-1}\right)$.

By Lemma 2.1, $\nabla f_{n}=\eta_{n} * \nabla f \in\left(L_{|x|^{1-N}}^{1}\right)^{N}$ and so, from the above,

$$
\left\|f_{n}(r, \cdot)-f_{n}(0)\right\|_{1, \mathbb{S}^{N-1}} \leq\left\||x|^{1-N}\left|\nabla f_{n}\right|\right\|_{1, B_{r}}
$$

In particular,

$$
\left|f _ { n } ( 0 ) \left\|\mathbb { S } ^ { N - 1 } \left|\leq\left\|f_{n}(r, \cdot)\right\|_{1, \mathbb{S}^{N-1}}+\left\||x|^{1-N}\left|\nabla f_{n}\right|\right\|_{1, B_{r}} .\right.\right.\right.
$$

The right-hand side is bounded since, as just noted, $f_{n}(r, \cdot) \rightarrow f(r, \cdot)$ in $L^{1}\left(\mathbb{S}^{N-1}\right)$ and since, by Lemma 2.1, $\nabla f_{n} \rightarrow \nabla f$ in $\left(L_{|x|^{1-N}}^{1}\right)^{N}$ (whence $\left\||x|^{1-N}\left|\nabla f_{n}\right|\right\|_{1, B_{r}} \rightarrow$ $\left.\left\||x|^{1-N}|\nabla f|\right\|_{1, B_{r}}\right)$. Thus, the sequence $f_{n}(0)$ is bounded. After passing to a subsequence, we may assume $\lim _{n \rightarrow \infty} f_{n}(0)=c \in \mathbb{R}$ and then (2.2) follows by letting $n \rightarrow \infty$ in $(2.3)$.

From the proof of Lemma 2.2, one should expect that 0 is a Lebesgue point of $f$ and that $c=f(0)$. This is shown, in a more general form, in Theorem 2.3 below.

If $f \in W_{l o c}^{1,1}$ and $z \in \mathbb{R}^{N}$, the convolution $\left(|x|^{1-N} *|\nabla f|\right)(z) \geq 0$ is unambiguously defined (but possibly $\infty$ ) by $\int_{\mathbb{R}^{N}}|z-y|^{1-N}|\nabla f(y)| d y=\int_{\mathbb{R}^{N}}|y|^{1-N}|\nabla f(z-y)| d y$.

Theorem 2.3. If $f \in W_{l o c}^{1,1}$ and if $z \in \mathbb{R}^{N}$ is such that $\left(|x|^{1-N} *|\nabla f|\right)(z)<\infty$, then $z$ is a Lebesgue point of $f$. Furthermore (recall (1.1)),

$$
\begin{aligned}
\left|B_{\rho}\right|^{-1} \int_{B_{\rho}(z)}|f(x)-f(z)| d x & \leq\left|\mathbb{S}^{N-1}\right|^{-1}\left\||x-z|^{1-N}|\nabla f|\right\|_{1, B_{\rho}(z)} \\
& \leq\left|\mathbb{S}^{N-1}\right|^{-1}\left(|x|^{1-N} *|\nabla f|\right)(z)
\end{aligned}
$$

for every $\rho>0$.

Proof. After replacing $f$ with $f_{z}:=f(\cdot+z)$, we may and shall assume $z=0$. The assumption $\left(|x|^{1-N} *|\nabla f|\right)(0)<\infty$ is exactly $\nabla f \in\left(L_{|x|^{1-N}}^{1}\right)^{N}$. Thus, by Lemma 2.2, there is a constant $c$ such that $\|f(r, \cdot)-c\|_{1, \mathbb{S}^{N-1}} \leq\left\||x|^{1-N} \nabla f\right\|_{1, B_{r}}$ for every $r>0$. 
Fix $\quad \rho>0$. Then, $\quad\|f(r, \cdot)-c\|_{1, \mathbb{S}^{N-1}} \leq\left\||x|^{1-N} \nabla f\right\|_{1, B_{\rho}} \quad$ when $\quad 0<r \leq \rho$, whence $\int_{B_{\rho}}|f(x)-c| d x \leq N^{-1} \rho^{N}\left\||x|^{1-N} \nabla f\right\|_{1, B_{\rho}}$, that is

$$
\left|B_{\rho}\right|^{-1} \int_{B_{\rho}}|f(x)-c| d x \leq\left|\mathbb{S}^{N-1}\right|^{-1}\left\||x|^{1-N} \nabla f\right\|_{1, B_{\rho}} .
$$

Since the right-hand side tends to 0 as $\rho \rightarrow 0$, it follows that 0 is a Lebesgue point of $f$ and (by (1.1)) that $c=f(0)$. Also, (2.4) (with $z=0$ ) follows from (2.5).

By different arguments, it is shown in Ziemer [32, p. 115] that the Lebesgue averages $\left|B_{\rho}\right|^{-1} \int_{B_{\rho}(z)} f(x) d x$ converge when $\left(|x|^{1-N} *|\nabla f|\right)(z)<\infty$, but this is weaker than saying that $z$ is a Lebesgue point of $f$.

The next main preliminary result is a simpler variant of [22, Theorem 2.1].

Lemma 2.4. Given $f \in L_{\text {loc }}^{1}$, suppose that for some subset $S \subset \mathbb{R}^{N}$ there are $0<\rho_{S} \leq \infty$ and a constant $C_{S}>0$ such that

$$
\left|B_{\rho}\right|^{-1} \int_{B_{\rho}(z)}|f(x)-f(z)| d x \leq C_{S},
$$

for every $z \in S$ and every $0<\rho<\rho_{S}$. Then,

$$
|f(y)-f(z)| \leq\left(1+2^{N}\right) C_{S},
$$

for every $y, z \in S$ with $|y-z|<\rho_{S} / 2$.

Proof. Evidently, it suffices to prove (2.7) when $y \neq z$. Let then $y, z \in S$ be such that $0<|y-z|<\rho_{S} / 2$. For every $\rho>0$,

$$
\begin{aligned}
|f(y)-f(z)| & =\left|B_{\rho}\right|^{-1} \int_{B_{\rho}(z)}|f(y)-f(z)| d x \\
& \leq\left|B_{\rho}\right|^{-1} \int_{B_{\rho}(z)}|f(x)-f(y)| d x+\left|B_{\rho}\right|^{-1} \int_{B_{\rho}(z)}|f(x)-f(z)| d x .
\end{aligned}
$$

Hence, by (2.6), $|f(y)-f(z)| \leq\left|B_{\rho}\right|^{-1} \int_{B_{\rho}(z)}|f(x)-f(y)| d x+C_{S}$ if $0<\rho<\rho_{S}$. In particular, if $\rho=|y-z|<\rho_{S} / 2$, then $B_{\rho}(z) \subset B_{2 \rho}(y)$ and so $\left|B_{\rho}\right|^{-1} \int_{B_{\rho}(z)}|f(x)-f(y)| d x \leq$ $2^{N}\left|B_{2 \rho}\right|^{-1} \int_{B_{2 \rho}(y)}|f(x)-f(y)| d x$, where $\left|B_{2 \rho}\right|=2^{N}\left|B_{\rho}\right|$ was used. Since $2 \rho<\rho_{S}$, (2.6) yields $\left|B_{2 \rho}\right|^{-1} \int_{B_{2 \rho}(y)}|f(x)-f(y)| d x \leq C_{S}$ and so $|f(y)-f(z)| \leq\left(1+2^{N}\right) C_{S}$.

Proof of Theorem 1.1. (i) First, $\left(|x|^{1-N} *|\nabla f|\right)(z) \leq\left\||x|^{1-N} *|\nabla f|\right\|_{\infty}$ for every $z \in \mathbb{R}^{N}$. Indeed, since this holds for a.e. $z \in \mathbb{R}^{N}$, any $z \in \mathbb{R}^{N}$ can be approximated by a sequence $z_{n}$ such that $\left(|x|^{1-N_{*}}|\nabla f|\right)\left(z_{n}\right) \leq\left\||x|^{1-N_{*}}|\nabla f|\right\|_{\infty}$ and the result follows from $\left(|x|^{1-N} *|\nabla f|\right)\left(z_{n}\right)=\int_{\mathbb{R}^{N}}\left|x-z_{n}\right|^{1-N}|\nabla f(x)| d x$ and Fatou's lemma. 
Recall that $\nabla f \in\left(L_{l o c}^{1}\right)^{N}$ if and only if $f \in W_{l o c}^{1,1}$. Hence, by Theorem 2.3, every $z \in \mathbb{R}^{N}$ is a Lebesgue point of $f$ and the inequality $\left|B_{\rho}\right|^{-1} \int_{B_{\rho}(z)}|f(x)-f(z)| d x \leq$ $\left|\mathbb{S}^{N-1}\right|^{-1}\left\||x|^{1-N} *|\nabla f|\right\|_{\infty}$ holds for every $\rho>0$. Therefore, with $S=\mathbb{R}^{N}, \rho_{S}=\infty$ and $C_{S}=\left|\mathbb{S}^{N-1}\right|^{-1}|||x|^{1-N_{*}}|\nabla f| \|_{\infty}$ in Lemma 2.4, we get $|f(y)-f(z)| \leq C \||x|^{1-N_{*}}$ $|\nabla f| \|_{\infty}$ for every $y, z \in \mathbb{R}^{N}$, where $C:=\left(1+2^{N}\right)\left|\mathbb{S}^{N-1}\right|^{-1}$. Thus,

$$
|f(y)| \leq|f(z)|+C|||x|^{1-N} *|\nabla f| \|_{\infty},
$$

for every $y, z \in \mathbb{R}^{N}$ and so sup $|f| \leq \inf |f|+C\left\||x|^{1-N} *|\nabla f|\right\|_{\infty}$. It is a simple exercise to check that since every point is a Lebesgue point of $f$, hence also of $|f|$, ess sup $|f|=\sup |f|$. This proves (1.2).

(ii) It is well-known that the Riesz potential $I_{1}$ is bounded from $L^{N, 1}$ into $L^{\infty}$ ([2, p. 228]). Thus, part (i) is applicable and (1.2) yields sup $|f|=\|f\|_{\infty} \leq$ $\inf |f|+C\|\nabla f\|_{N, 1}$ with $C>0$ independent of $f$. To prove the uniform continuity of $f$, recall first that $|x|^{1-N} \in L^{N /(N-1), \infty}$, the associated space of $L^{N, 1}$. Thus, by Hölder's inequality ([2, p. 60]),

$$
\begin{aligned}
\left\||x-z|^{1-N} \nabla f\right\|_{1, B_{\rho}(z)} & \leq\left\||x-z|^{1-N}\right\|_{N /(N-1), \infty, B_{\rho}(z)}\|\nabla f\|_{N, 1, B_{\rho}(z)} \\
& =\left\||x|^{1-N}\right\|_{N /(N-1), \infty, B_{\rho}}\|\nabla f\|_{N, 1, B_{\rho}(z)} \\
& \leq\left\||x|^{1-N}\right\|_{N /(N-1), \infty}\|\nabla f\|_{N, 1, B_{\rho}(z)}
\end{aligned}
$$

for every $z \in \mathbb{R}^{N}$ and every $\rho>0$.

Since $\left|B_{\rho}(z)\right|=\left|B_{\rho}\right|$ is independent of $z$, it follows from the absolute continuity of the $L^{N, 1}$ norm $\left(\left[2\right.\right.$, p. 222]) that $\lim _{\rho \rightarrow 0}\|\nabla f\|_{N, 1, B_{\rho}(z)}=0$ uniformly for $z \in \mathbb{R}^{N}$. Accordingly, given $\varepsilon>0$, choose $\rho_{\varepsilon}>0$ such that $\|\nabla f\|_{N, 1, B_{\rho}(z)}<\varepsilon$ for every $z \in \mathbb{R}^{N}$ and every $\rho<\rho_{\varepsilon}$. From the above and from the first inequality in (2.4), $\left|B_{\rho}\right|^{-1} \int_{B_{\rho}(z)}|f(x)-f(z)| d x \leq C \varepsilon$ for every $z \in \mathbb{R}^{N}$ and every $\rho<\rho_{\varepsilon}$, where $C:=$ $\left|\mathbb{S}^{N-1}\right|^{-1}\left\||x|^{1-N}\right\|_{N /(N-1), \infty}$. Thus, with $S=\mathbb{R}^{N}, \rho_{S}=\rho_{\varepsilon}$ and $C_{S}=C \varepsilon$ in Lemma 2.4, it follows that $|f(y)-f(z)| \leq\left(1+2^{N}\right) C \varepsilon$ whenever $y, z \in \mathbb{R}^{N}$ and $|y-z|<\rho_{\varepsilon} / 2$. This completes the proof.

Remark 2.5. The proof of the uniform continuity in Theorem 1.1 (ii) uses only $|x|^{1-N_{*}} *|\nabla f| \in L^{\infty}$ and $\lim _{\rho \rightarrow 0}\|\nabla f\|_{N, 1, B_{\rho}(z)}=0$ uniformly for $z \in \mathbb{R}^{N}$. This will be used in the proof of Theorem 4.2.

By a cut-off argument, $\left({ }^{1}\right)$ the uniform continuity of $f$ when $\nabla f \in\left(L^{N, 1}\right)^{N}$ proves again its continuity when $\nabla f \in\left(L_{l o c}^{N, 1}\right)^{N}$. In contrast, there are obstacles to tweaking Stein's proof in [26] to obtain the uniform continuity when $\nabla f \in\left(L^{N, 1}\right)^{N}$. The main difficulty is to reduce the problem to the case when $f=I_{1}\left(\sum_{j=1}^{N} R_{j} \partial_{j} f\right)$ where the

$\left({ }^{1}\right)$ Justified by Lemma 3.4 later. 
$R_{j}$ are the Riesz transforms. At best, this can only hold after modifying $f$ by a constant (for the local result, it may be assumed that Supp $f$ is compact and then the formula follows from its validity when $\left.f \in \mathcal{C}_{0}^{\infty}\right)$. In spirit, the simpler proof in DeVore and Sharpley [6] is close to the one given above and it could easily be complemented to yield the uniform continuity. However, it is not complete because a would-be routine passage to the limit cannot be justified without the nontrivial Lemma 2.1.

\section{Behavior at infinity}

Both parts (i) and (ii) of Theorem 1.1 can be complemented by showing that, in a sense to be specified, $f$ has a finite constant limit at infinity. The first part of the proof of Theorem 3.1 (i) below parallels that of [21, Theorem 3.2], with some necessary technical differences.

Theorem 3.1. Assume $N>1$.

(i) If $\nabla f \in\left(L_{\text {loc }}^{1}\right)^{N}$ and $|x|^{1-N} *|\nabla f| \in L^{\infty}$, there is a unique constant $c_{f} \in \mathbb{R}$ such that, for every $z \in \mathbb{R}^{N}$,

$$
\lim _{r \rightarrow \infty} f(z+r \omega)=c_{f}
$$

for a.e. $\omega \in \mathbb{S}^{N-1}$ (depending upon $z$ ). In particular, if $f$ is radially symmetric, then $\lim _{|x| \rightarrow \infty} f(x)=c_{f}$ and, in addition, $\left\|f-c_{f}\right\|_{\infty} \leq\left|\mathbb{S}^{N-1}\right|^{-1}\left\||x|^{1-N} *|\nabla f|\right\|_{\infty}$.

(ii) If, in (i), $f$ is uniformly continuous, then (3.1) holds for a.e. $\omega \in \mathbb{S}^{N-1}$ and every $z \in \mathbb{R}^{N}$ (case in point: $\omega$ is now independent of $z$ ).

Proof. (i) The proof consists in showing that (3.1) holds with $c_{f}=c_{f, z}$ possibly depending upon $z$ and, next, that $c_{f, z}$ is independent of $z$. We henceforth drop the subscript $f$ for simplicity, i.e., $c_{f, z}:=c_{z}$. By Theorem $1.1(\mathrm{i}),\left(|x|^{1-N_{*}} *|\nabla f|\right)(z)<\infty$ i.e., $\nabla f_{z} \in\left(L_{|x|^{1-N}}^{1}\right)^{N}$ where $f_{z}:=f(\cdot+z)$. Thus, it suffices to prove the existence of $c_{z}$ when $z=0$. As before, we use the notation $f(r, \omega)$ for $f(r \omega)$ when $r \geq 0$ and $\omega \in \mathbb{S}^{N-1}$.

As noted at the beginning of the proof of Lemma 2.2, $f \in W_{l o c}^{1,1}$. In particular, $f$ is locally absolutely continuous on almost every line parallel to the coordinate axis $x_{j}$ and, on such lines, the classical and weak derivatives $\partial_{j} f$ coincide. Since the measures $d r$ and $r^{N-1} d r$ are equivalent away from the origin, this implies that, after passing to spherical coordinates, $f \in W_{l o c}^{1,1}\left((0, \infty) \times \mathbb{S}^{N-1}\right)$ and so $f(\cdot, \omega)$ is locally absolutely continuous on $(0, \infty)$ for a.e. $\omega \in \mathbb{S}^{N-1}$, with classical radial derivative $\partial_{r} f(r, \omega)=\nabla f(r, \omega) \cdot \omega$. 
By Fubini's theorem in spherical coordinates (use $\left.|x|^{1-N}|\nabla f| \in L^{1}\right),|\nabla f(\cdot, \omega)| \in$ $L^{1}(0, \infty)$ for a.e. $\omega \in \mathbb{S}^{N-1}$, whence $\partial_{r} f(\cdot, \omega) \in L^{1}(0, \infty)$ for a.e. $\omega \in \mathbb{S}^{N-1}$. Therefore,

$$
g(r, \omega):=\int_{\infty}^{r} \partial_{r} f(t, \omega) d t
$$

is a.e. defined and measurable on $(0, \infty) \times \mathbb{S}^{N-1}$. For a.e. $\omega \in \mathbb{S}^{N-1}$, the function $g(\cdot, \omega)$ is locally absolutely continuous and a.e. differentiable on $(0, \infty)$ with $\partial_{r} g(\cdot, \omega)=\partial_{r} f(\cdot, \omega)$ and

$$
c_{0}(\omega):=f(\cdot, \omega)-g(\cdot, \omega)
$$

is a function independent of $r>0$ since both $f(\cdot, \omega)$ and $g(\cdot, \omega)$ are locally absolutely continuous with the same a.e. derivative. Also, $\lim _{r \rightarrow \infty} g(r, \omega)=0$, which is (3.1) when $z=0$, but with $c_{f}=c_{0}(\omega)$. The remainder of the proof (when $z=0$ ) consists in showing that $c_{0}$ is constant.

Observe that $g(r, \cdot) \in L^{1}\left(\mathbb{S}^{N-1}\right)$ for every $r>0$ and that $\lim _{r \rightarrow \infty}\|g(r, \cdot)\|_{1, \mathbb{S}^{N-1}} \leq$ $\lim _{r \rightarrow \infty} \int_{\mathbb{R}^{N} \backslash B_{r}}|x|^{1-N}|\nabla f|=0$. Thus, by $(3.2)$,

$$
\lim _{r \rightarrow \infty}\left\|f(r, \cdot)-c_{0}\right\|_{1, \mathbb{S}^{N-1}}=0 .
$$

Next, we need the Poincaré-Wirtinger inequality on the sphere $\mathbb{S}^{N-1}$ : Since $N>1$, the unit sphere is connected and so

$$
\|w-\bar{w}\|_{1, \mathbb{S}^{N-1}} \leq C\left\|\nabla_{\mathbb{S}^{N-1}} w\right\|_{1, \mathbb{S}^{N-1}}
$$

for every $w \in W^{1,1}\left(\mathbb{S}^{N-1}\right)$, where $\nabla_{\mathbb{S}^{N-1}}$ is the gradient of $w$ for the natural Riemannian structure of the unit sphere, $C>0$ is a constant independent of $w$ and $\bar{w}$ is the average of $w$ on $\mathbb{S}^{N-1}$. The Poincaré-Wirtinger inequality on the sphere is well documented mostly when $W^{1,1}\left(\mathbb{S}^{N-1}\right)$ is replaced with $W^{1,2}\left(\mathbb{S}^{N-1}\right)$ ([19]) but a proof valid in $W^{1, p}\left(\mathbb{S}^{N-1}\right)$ for any $1 \leq p \leq \infty$ follows, by the usual contradiction argument, from the connectedness of $\mathbb{S}^{N-1}$ and from the compactness of the embedding $W^{1, p}\left(\mathbb{S}^{N-1}\right) \hookrightarrow L^{p}\left(\mathbb{S}^{N-1}\right)$.

Assume $f \in \mathcal{C}^{\infty}$. If $r>0$ is fixed, then $\nabla_{\mathbb{S}^{N-1}} f(r, \omega)=r P_{\omega} \nabla f(r, \omega)$, where $P_{\omega}$ is the orthogonal projection on the tangent space $\{\omega\}^{\perp}$ of $\mathbb{S}^{N-1}$ at $\omega$, whence $\left|\nabla_{\mathbb{S}^{N-1}} f(r, \omega)\right| \leq r|\nabla f(r, \omega)|$. Thus, with $\bar{f}(r)$ denoting the average of $f(r, \cdot)$ on $\mathbb{S}^{N-1}$, it follows from $(3.4)$ that $\|f(r, \cdot)-\bar{f}(r)\|_{1, \mathbb{S}^{N-1}} \leq C r\|\nabla f(r, \cdot)\|_{1, \mathbb{S}^{N-1}}$ and so

$$
\begin{aligned}
& \int_{0}^{\infty} r^{-1}\|f(r, \cdot)-\bar{f}(r)\|_{1, \mathbb{S}^{N-1}} d r \\
& \quad \leq C \int_{0}^{\infty}\|\nabla f(r, \cdot)\|_{1, \mathbb{S}^{N-1}} d r=C \int_{\mathbb{R}^{N}}|x|^{1-N}|\nabla f|<\infty .
\end{aligned}
$$


The above inequality continues to hold without the extra assumption that $f \in \mathcal{C}^{\infty}$. Indeed, if $f_{n}:=\eta_{n} * f \in \mathcal{C}^{\infty}$ with $\eta_{n}$ the mollifying sequence of Lemma 2.1, then $\int_{\mathbb{R}^{N}}|x|^{1-N}\left|\nabla f_{n}\right| \rightarrow \int_{\mathbb{R}^{N}}|x|^{1-N}|\nabla f|$ and, since also $f_{n} \rightarrow f$ in $W_{l o c}^{1,1}$, it follows that $f_{n}(r, \cdot) \rightarrow f(r, \cdot)$ in $L^{1}\left(\mathbb{S}^{N-1}\right)$, so that $\bar{f}_{n}(r) \rightarrow \bar{f}(r)$. Thus, $r^{-1}\left\|f_{n}(r, \cdot)-\bar{f}_{n}(r)\right\|_{1, \mathbb{S}^{N-1}}$ $\rightarrow r^{-1}\|f(r, \cdot)-\bar{f}(r)\|_{1, \mathbb{S}^{N-1}}$ for every $r>0$ and (3.5) follows from the same inequality for $f_{n}$ and from Fatou's lemma.

Since the left-hand side of (3.5) is finite and $r^{-1}$ is not integrable at infinity, there is a sequence $r_{n} \rightarrow \infty$ such that $\lim \left\|f\left(r_{n}, \cdot\right)-\bar{f}\left(r_{n}\right)\right\|_{1, \mathbb{S}^{N-1}}=0$. By (3.3), $\lim \left\|f\left(r_{n}, \cdot\right)-c_{0}\right\|_{1, \mathbb{S}^{N-1}}=0$. Thus, $\lim \left\|\bar{f}\left(r_{n}\right)-c_{0}\right\|_{1, \mathbb{S}^{N-1}}=0$ and, since $\bar{f}\left(r_{n}\right)$ is independent of $\omega$, it follows that $c_{0}$ is constant. This proves (3.1) with $c_{f}=c_{z}$ when $z=0$ and, hence, in general.

If (3.1) had been proved with $c_{f}=c_{z}$ for every $\omega \in \mathbb{S}^{N-1}$ and every $z \in \mathbb{R}^{N}$, the limit along the ray from 0 to $z$ would have to be both $c_{0}$ and $c_{z}$, thereby showing that $c_{z}$ is independent of $z$. This elementary argument cannot be used with the weaker "a.e. $\omega$ " property and the proof that $c_{z}=c_{0}$ is more subtle.

If $R>\rho>0$, let $\Omega_{R, \rho}(z)$ denote the annulus

$$
\Omega_{R, \rho}(z):=\left\{x \in \mathbb{R}^{N}: R-\rho<|x-z|<R+\rho\right\} .
$$

Note that if $z$ and $\rho$ are held fixed,

$$
\lim _{R \rightarrow \infty}\left\||x-z|^{1-N}\left(f-c_{z}\right)\right\|_{1, \Omega_{R, \rho}(z)}=0 .
$$

This follows from $\left\||x-z|^{1-N}\left(f-c_{z}\right)\right\|_{1, \Omega_{R, \rho}(z)}=\left\||x|^{1-N}\left(f_{z}-c_{z}\right)\right\|_{1, \Omega_{R, \rho}(0)} \leq$ $2 \rho \sup _{r>R-\rho}\left\|f_{z}(r, \cdot)-c_{z}\right\|_{1, \mathbb{S}^{N-1}}$ and from $\sup _{r>R-\rho}\left\|f_{z}(r, \cdot)-c_{z}\right\|_{1, \mathbb{S}^{N-1}} \rightarrow 0$ when $R \rightarrow \infty$ since $\lim _{r \rightarrow \infty}\left\|f_{z}(r, \cdot)-c_{z}\right\|_{1, \mathbb{S}^{N-1}}=0$ by $(3.3)$.

In particular, with $z$ fixed and $\rho=|z|+1$,

$$
\lim _{R \rightarrow \infty}\left\||x-z|^{1-N}\left(f-c_{z}\right)\right\|_{1, \Omega_{R,|z|+1}(z)}=0
$$

and, with $z=0$ and $\rho=1$,

$$
\lim _{R \rightarrow \infty}\left\||x|^{1-N}\left(f-c_{0}\right)\right\|_{1, \Omega_{R, 1}(0)}=0 .
$$

Now, observe that $\Omega_{R, 1}(0) \subset \Omega_{R,|z|+1}(z)$. Hence, by (3.6), $\lim _{R \rightarrow \infty} \||x-z|^{1-N}(f-$ $\left.c_{z}\right) \|_{1, \Omega_{R, 1}(0)}=0$. Next, $|x|^{1-N}<2^{N-1}|x-z|^{1-N}$ if $x \in \Omega_{R, 1}(0)$ and $R \geq|z|+1$ and so $\lim _{R \rightarrow \infty}\left\||x|^{1-N}\left(f-c_{z}\right)\right\|_{1, \Omega_{R, 1}(0)}=0$. Together with (3.7), it follows that $\lim _{R \rightarrow \infty}\left\||x|^{1-N}\left(c_{z}-c_{0}\right)\right\|_{1, \Omega_{R, 1}(0)}=0$, which can only happen if $c_{z}=c_{0}$ because $\left\||x|^{1-N}\left(c_{z}-c_{0}\right)\right\|_{1, \Omega_{R, 1}(0)}=2\left|\mathbb{S}^{N-1} \| c_{z}-c_{0}\right|$ is independent of $R$.

To complete the proof of (i), let $f$ be radially symmetric. It is plain that (3.3) (with $c_{0}=c_{f}$ ) amounts to $\lim _{|x| \rightarrow \infty} f(x)-c_{f}=0$. Also, recall that, in general, (3.3) 
follows from $\left\|f(r, \cdot)-c_{f}\right\|_{1, \mathbb{S}^{N-1}} \leq \int_{\mathbb{R}^{N} \backslash B_{r}}|x|^{1-N}|\nabla f|$ for every $r>0$. The right-hand side is majorized by $\int_{\mathbb{R}^{N} \backslash B_{r}}|x|^{1-N}|\nabla f| \leq\left\||x|^{1-N} *|\nabla f|\right\|_{\infty}$ and, under the radially symmetric assumption, the left-hand side is $\left|\mathbb{S}^{N-1}\right|\left|f(y)-c_{f}\right|$ for every $y$ such that $|y|=r>0$. Therefore, $\left|f(y)-c_{f}\right| \leq\left|\mathbb{S}^{N-1}\right|^{-1}\left\||x|^{1-N} *|\nabla f|\right\|_{\infty}$ for every $y \neq 0$ and so $\left\|f-c_{f}\right\|_{\infty} \leq\left|\mathbb{S}^{N-1}\right|^{-1}\left\||x|^{1-N_{*}} *|\nabla f|\right\|_{\infty}$.

(ii) Let $\left(z_{n}\right)$ be a countable dense subset of $\mathbb{R}^{N}$. By (i), there is a subset $E \subset \mathbb{S}^{N-1}$ with $\mathbb{S}^{N-1}$-measure 0 such that $\lim _{r \rightarrow \infty} f\left(z_{n}+r \omega\right)=c_{f}$ for every $n \in \mathbb{N}$ and every $\omega \in \mathbb{S}^{N-1} \backslash E$. Choose $\omega \in \mathbb{S}^{N-1} \backslash E$ and $z \in \mathbb{R}^{N}$ and, given $\varepsilon>0$, let $\delta>0$ be such that $|f(x)-f(y)|<\varepsilon / 2$ whenever $|x-y|<\delta$. Choose $n$ such that $\left|z_{n}-z\right|<\delta$. If $r>0$ is large enough, then $\left|f\left(z_{n}+r \omega\right)-c_{f}\right|<\varepsilon / 2$. Since $\left|\left(z_{n}+r \omega\right)-(z+r \omega)\right|=\left|z_{n}-z\right|<\delta$, it follows that $\left|f(z+r \omega)-c_{f}\right|<\varepsilon$. Thus, $\lim _{r \rightarrow \infty} f(z+r \omega)=c_{f}$.

Remark 3.2. From the above proof, $c_{f}=\lim _{r \rightarrow \infty} \int_{\mathbb{S}^{N-1}} f(r, \omega) d \omega$. By elementary arguments, this implies $c_{f}=\lim _{r \rightarrow \infty}\left|B_{r}\right|^{-1} \int_{B_{r}} f$.

A function $f \in \mathcal{B U C}$ satisfying (3.1) for a.e. $\omega \in \mathbb{S}^{N-1}$ and every $z \in \mathbb{R}^{N}$ need not tend uniformly to $c_{f}$. An example with $N=2$ and $c_{f}=0$ is $f\left(x_{1}, x_{2}\right)=\varphi\left(x_{1}\right)$ with $\varphi \in \mathcal{C}_{0}^{\infty}(\mathbb{R})$. More sophisticated examples can be found with $|x|^{1-N} *|\nabla f| \in L^{\infty}$ and even with (3.1) holding for every $\omega \in \mathbb{S}^{N-1}$ and every $z \in \mathbb{R}^{N}$. Thus, in Theorem 3.1, $c_{f}$ need not be the uniform limit of $f$ at infinity.

If $N=1$, Theorem 3.1 is false because $f$ may tend to two different limits as $x \rightarrow \pm \infty$. A result closely related to Theorem 3.1 (i) is given in Mizuta [16, Theorem 2] under the assumption $\nabla f \in\left(L^{p}\right)^{N}$ with $1<p<N$, neither weaker nor stronger than $|x|^{1-N_{*}} *|\nabla f| \in L^{\infty}$. Mizuta also proves $\left|f_{z}(r, \omega)-c_{z}\right|=o\left(r^{1-N / p}\right)$ but does not address the question whether $c_{z}$ is independent of $z$.

We now proceed to showing that $\lim _{|x| \rightarrow \infty} f(x)=c_{f}$ if $N>1$ and $\nabla f \in\left(L^{N, 1}\right)^{N}$. We were unable to deduce this from Theorem 3.1 and will give a direct proof. To do this, we need several preliminary technical results which are all well-known in classical Sobolev spaces, but harder to find in the Lorentz-Sobolev setting. We refrain from discussing anything significantly more general than needed in this paper. If $\Omega$ is an open subset of $\mathbb{R}^{N}$ and $1 \leq q<\infty$, we set

$$
W^{1} L^{q, 1}(\Omega):=\left\{h \in L^{q, 1}(\Omega): \nabla h \in\left(L^{q, 1}(\Omega)\right)^{N}\right\},
$$

a Banach space for the norm $\|f\|_{W^{1} L^{q, 1}(\Omega)}:=\|f\|_{q, 1, \Omega}+\|\nabla f\|_{q, 1, \Omega}$. When $\Omega=\mathbb{R}^{N}$, we simply use $W^{1} L^{q, 1}$, with norm $\|f\|_{W^{1} L^{q, 1}}$.

Lemma 3.3. If $\Omega$ is bounded with a Lipschitz boundary, there is a bounded linear extension operator $E: W^{1} L^{q, 1}(\Omega) \rightarrow W^{1} L^{q, 1}$. 
Proof. Such an extension operator exists that maps $W^{1,1}(\Omega)$ into $W^{1,1}$ and $W^{1, \infty}(\Omega)$ into $W^{1, \infty}([25$, Theorem 5, p. 181]). By interpolation (see DeVore and Scherer [7, Theorem 2]), $E$ also maps $W^{1} L^{q, 1}(\Omega)$ into $W^{1} L^{q, 1}$ when $1<q<\infty$.

Lemma 3.4. If $\Omega$ is bounded with Lipschitz boundary and $\nabla h \in\left(L^{q, 1}(\Omega)\right)^{N}$, then $h \in W^{1} L^{q, 1}(\Omega)$.

Proof. Since $L^{q, 1}(\Omega) \subset L^{q}(\Omega)$, it follows that $\nabla h \in\left(L^{q}(\Omega)\right)^{N}$, whence $h \in W^{1, q}(\Omega)$ by [15, p. 23]. But $W^{1, q}(\Omega) \subset L^{p}(\Omega)$ for some $p>q$ and $L^{p}(\Omega) \subset L^{q, 1}(\Omega)$ since $\Omega$ is bounded. Thus, $h \in L^{q, 1}(\Omega)$ and so $f \in W^{1} L^{q, 1}(\Omega)$.

The next lemma is a Poincaré-Wirtinger inequality in $W^{1} L^{q, 1}(\Omega)$.

Lemma 3.5. Suppose that $\Omega$ is bounded and connected with Lipschitz boundary.

(i) There is a constant $C>0$ such that $\|h\|_{W^{1} L^{q, 1}(\Omega)} \leq C\|\nabla h\|_{q, 1, \Omega}$ for every $h \in$ $W^{1} L^{q, 1}(\Omega)$ such that $\int_{\Omega} h=0$.

(ii) If $1 \leq q<N$ and if $\nabla h \in\left(L^{q, 1}(\Omega)\right)^{N}$, then $h \in L^{q^{*}, 1}(\Omega)$ and there is a constant $C>0$ independent of $h$ such that $\|h-\bar{h}\|_{q^{*}, 1, \Omega} \leq C\|\nabla h\|_{q, 1, \Omega}$, where $\bar{h}:=|\Omega|^{-1} \int_{\Omega} h$.

Proof. (i) The embedding $W^{1} L^{q, 1}(\Omega) \hookrightarrow L^{q, 1}(\Omega)$ is compact. Indeed, it is the composite $W^{1} L^{q, 1}(\Omega) \hookrightarrow W^{1, q}(\Omega)$ (continuous), followed by $W^{1, q}(\Omega) \hookrightarrow L^{p}(\Omega)$ with $p>q$ sufficiently close to $q$ (compact), followed by $L^{p}(\Omega) \hookrightarrow L^{q, 1}(\Omega)$ (continuous). The result then follows by a standard contradiction argument.

(ii) Choose $p \in(q, N)$. The identity operator is bounded from $W^{1,1}(\Omega)$ into $L^{1^{*}}(\Omega)$ and from $W^{1, p}(\Omega)$ into $L^{p^{*}}(\Omega)$. By [7, Theorem 2], it is therefore bounded from $W^{1} L^{q, 1}(\Omega)$ into $L^{q^{*}, 1}(\Omega)$ if $q>1$. The same thing is true if $q=1$; see for instance Talenti [27, Theorem 4.A]. Thus, by (i), there is a constant $C>0$ such that $\|h\|_{q^{*}, 1, \Omega} \leq C\|\nabla h\|_{q, 1, \Omega}$ for every $h \in W^{1} L^{q, 1}(\Omega)$ such that $\int_{\Omega} h=0$. By Lemma 3.4, this inequality holds if it is only assumed that $\nabla h \in\left(L^{q, 1}(\Omega)\right)^{N}$ and the condition $\int_{\Omega} h=0$ can be dropped after replacing $h$ with $h-\bar{h}$.

Results in the spirit of Lemmas 3.4 and 3.5 go back to the early days of interpolation theory; see notably O'Neil [18] or Peetre [20]. For example, when $\Omega=\mathbb{R}^{N}$ and $1<q<N$, it follows from [20, Theorem 8.1] that inf $\operatorname{in}_{c \in \mathbb{R}}\|h-c\|_{q^{*}, 1} \leq C\|\nabla h\|_{q, 1}$ where $C>0$ is independent of $h$ with $\nabla h \in\left(L^{q, 1}\right)^{N}$. This inequality is closely related to Lemma 3.5 (ii).

The last preliminary lemma relies on a scaling property. For every $\rho>0$, we denote $\Omega_{\rho}$ the annulus

$$
\Omega_{\rho}:=\left\{x \in \mathbb{R}^{N}: \rho<|x|<2 \rho\right\}=\rho \Omega_{1} .
$$


Lemma 3.6. If $N>1$, there is a constant $C>0$ independent of $\rho>0$ such that

$$
\|f\|_{\infty, \Omega_{\rho}} \leq C\|\nabla f\|_{N, 1, \Omega_{\rho}}
$$

for every $f \in \mathcal{D}^{\prime}\left(\Omega_{\rho}\right)$ with $\nabla f \in\left(L^{N, 1}\left(\Omega_{\rho}\right)\right)^{N}$ and $\int_{\Omega_{\rho}} f=0$ (this makes sense since, by Lemma 3.4, $\left.f \in W^{1} L^{N, 1}\left(\Omega_{\rho}\right) \subset L^{1}\left(\Omega_{\rho}\right)\right)$.

Proof. We begin with the case $\rho=1$. By Lemma 3.4, $f \in W^{1} L^{N, 1}\left(\Omega_{1}\right)$ and so, by Lemma 3.3 , there is $\tilde{f} \in W^{1} L^{N, 1}$ such that $\tilde{f}=f$ on $\Omega_{1}$ and $\|\tilde{f}\|_{W^{1} L^{N, 1}} \leq$ $K\|f\|_{W^{1} L^{N, 1}\left(\Omega_{1}\right)}$ where $K>0$ is independent of $f$.

Next, by Theorem 1.1 (ii), $\tilde{f}$ (hence $f$ ) is continuous and $\|f\|_{\infty, \Omega_{1}}=\sup |\tilde{f}| \leq$ $\inf |\tilde{f}|+C\|\nabla \tilde{f}\|_{N, 1}$ with $C>0$ independent of $\tilde{f}$. Since $\int_{\Omega_{1}} f=0$ and $f$ is continuous, $f$ (hence $\tilde{f}$ ) must vanish at some point of $\Omega_{1}$, whence inf $|\tilde{f}|=0$. Furthermore, since $\|\nabla \tilde{f}\|_{N, 1} \leq\|\tilde{f}\|_{W^{1} L^{N, 1}} \leq K\|f\|_{W^{1} L^{N, 1}\left(\Omega_{1}\right)}$, we infer that $\|f\|_{\infty, \Omega_{1}} \leq C\|f\|_{W^{1} L^{N, 1}\left(\Omega_{1}\right)}$ after changing $C K$ into $C$. Then, by Lemma 3.5 (i) (since $\Omega_{1}$ is connected when $N>1)$

$$
\|f\|_{\infty, \Omega_{1}} \leq C\|\nabla f\|_{N, 1, \Omega_{1}},
$$

after a final modification of $C$ independent of $f$. This proves (3.9) when $\rho=1$.

The general case $\rho>0$ follows by scaling. If $\nabla f \in\left(L^{N, 1}\left(\Omega_{\rho}\right)^{N}\right.$ and $\int_{\Omega_{\rho}} f=0$, set $f_{\rho}(x):=f(\rho x)$ for $x \in \Omega_{1}$, so that $\nabla f_{\rho}=\rho \nabla f(\rho \cdot) \in\left(L^{N, 1}\left(\Omega_{1}\right)^{N}\right.$ and $\int_{\Omega_{1}} f_{\rho}=0$. By (3.10), $\left\|f_{\rho}\right\|_{\infty, \Omega_{1}} \leq C\|\rho \nabla f(\rho \cdot)\|_{N, 1, \Omega_{1}}=C \rho\|\nabla f(\rho \cdot)\|_{N, 1, \Omega_{1}}$. Since it is plain that $\left\|f_{\rho}\right\|_{\infty, \Omega_{1}}=\|f\|_{\infty, \Omega_{\rho}}$, this reads $\|f\|_{\infty, \Omega_{\rho}} \leq C \rho\|\nabla f(\rho \cdot)\|_{N, 1, \Omega_{1}}$ and so it suffices to show that $\|\nabla f(\rho \cdot)\|_{N, 1, \Omega_{1}}=\rho^{-1}\|\nabla f\|_{N, 1, \Omega_{\rho}}$ or, more generally, that $\|h(\rho \cdot)\|_{N, 1, \Omega_{1}}=$ $\rho^{-1}\|h\|_{N, 1, \Omega_{\rho}}$ for every $h \in L^{N, 1}\left(\Omega_{\rho}\right)$. This follows at once from $h(\rho \cdot)^{*}(t)=h^{*}\left(\rho^{N} t\right)$ and from the definition of the $L^{N, 1}$ norms.

Theorem 3.7. If $N>1$ and $\nabla f \in\left(L^{N, 1}\right)^{N}$, there is a unique constant $c_{f} \in \mathbb{R}$ such that $\lim _{|x| \rightarrow \infty} f(x)=c_{f}$. Furthermore, there is a constant $C>0$ independent of $f$ such that $\left\|f-c_{f}\right\|_{\infty} \leq C\|\nabla f\|_{N, 1}$.

Proof. The uniqueness of $c_{f}$ is obvious. We focus on its existence. If $h \in L_{l o c}^{1}$, define the radial symmetrization $h_{\text {rad }}$ of $h$ by

$$
h_{\text {rad }}(x):=\left|\mathbb{S}^{N-1}\right|^{-1} \int_{\mathbb{S}^{N-1}} h(|x| \omega) d \omega .
$$

This definition makes sense since, by Fubini's theorem in spherical coordinates, $h(r, \cdot) \in L^{1}\left(\mathbb{S}^{N-1}\right)$ for a.e. $r>0$. Accordingly, $h_{\text {rad }}(x)$ is defined for $x$ in almost every sphere centered at the origin. Furthermore, it is readily checked that $h \mapsto h_{\text {rad }}$ is linear and continuous on $L^{p}$ for every $1 \leq p \leq \infty$. By interpolation, it remains linear and continuous on $L^{N, 1}$. 
If $f \in W_{l o c}^{1,1}$ (in particular, if $\left.\nabla f \in\left(L^{N, 1}\right)^{N}\right)$, it is shown in the proof of [21, Lemma 4.1] that $f_{\text {rad }} \in W_{l o c}^{1,1}$ with $\nabla f_{\text {rad }}=|x|^{-1}\left(\partial_{r} f\right)_{\text {rad }} x$. Since $\nabla f \in\left(L^{N, 1}\right)^{N}$ implies $\partial_{r} f=|x|^{-1} \nabla f \cdot x \in L^{N, 1}$, it follows from the above with $h=\partial_{r} f$ that $\nabla f_{\text {rad }} \in$ $\left(L^{N, 1}\right)^{N}$ and that $\left\|\nabla f_{\text {rad }}\right\|_{N, 1} \leq\|\nabla f\|_{N, 1}$.

In particular, $|x|^{1-N_{*}}\left|\nabla f_{\text {rad }}\right| \in L^{\infty}$. Obviously, $f_{\text {rad }}$ is radially symmetric, so that, by Theorem 3.1 (i), $f_{\text {rad }}(x)$ has a uniform finite constant limit $c_{f_{\text {rad }}}$ as $|x| \rightarrow$

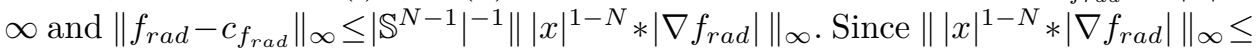
$C\left\|\nabla f_{\text {rad }}\right\|_{N, 1} \leq C\|\nabla f\|_{N, 1}$, it follows that $\left\|f_{\text {rad }}-c_{f_{\text {rad }}}\right\|_{\infty} \leq C\|\nabla f\|_{N, 1}$ after changing $\left|\mathbb{S}^{N-1}\right|^{-1} C$ into $C$.

We shall prove the theorem with $c_{f}=c_{f_{\text {rad }}}$. To do this, it suffices to show that $\lim _{|x| \rightarrow \infty}\left(f-f_{\text {rad }}\right)(x)=0$ and that $\left\|f-f_{\text {rad }}\right\|_{\infty} \leq C\|\nabla f\|_{N, 1}$ for some other constant $C$.

From the very definition of $f_{\text {rad }}$, it follows that $\int_{\mathbb{S}^{N-1}}\left(f-f_{\text {rad }}\right)(r, \omega) d \omega=0$ for a.e. $r>0$. Hence, $\int_{\Omega_{\rho}}\left(f-f_{\text {rad }}\right)=0$ for every $\rho>0$ (see (3.8)). Let $R>0$ be fixed. It is plain that $\left\|f-f_{\text {rad }}\right\|_{\infty, \mathbb{R}^{N} \backslash \bar{B}_{R}}=\sup _{\rho>R}\left\|f-f_{\text {rad }}\right\|_{\infty, \Omega_{\rho}}$. On the other hand, by Lemma 3.6,

$$
\left\|f-f_{\text {rad }}\right\|_{\infty, \Omega_{\rho}} \leq C\left\|\nabla\left(f-f_{\text {rad }}\right)\right\|_{N, 1, \Omega_{\rho}} \leq C\left\|\nabla\left(f-f_{\text {rad }}\right)\right\|_{N, 1, \mathbb{R}^{N} \backslash \bar{B}_{R}},
$$

for every $\rho>R$, where $C>0$ is independent of $R$ and $\rho$. Altogether, $\left\|f-f_{\text {rad }}\right\|_{\infty, \mathbb{R}^{N} \backslash \bar{B}_{R}} \leq C\left\|\nabla\left(f-f_{\text {rad }}\right)\right\|_{N, 1, \mathbb{R}^{N} \backslash \bar{B}_{R}} \quad$ (same $\left.C\right)$. Since $\nabla\left(f-f_{\text {rad }}\right) \in$ $\left(L^{N, 1}\right)^{N}$, it follows that $\lim _{R \rightarrow \infty}\left\|\nabla\left(f-f_{\text {rad }}\right)\right\|_{N, 1, \mathbb{R}^{N} \backslash \bar{B}_{R}}=0$. Consequently, $\lim _{R \rightarrow \infty}\left\|f-f_{\text {rad }}\right\|_{\infty, \mathbb{R}^{N} \backslash \bar{B}_{R}}=0$. By the continuity of $f-f_{\text {rad }}$, this amounts to $\lim _{|x| \rightarrow \infty}\left(f-f_{\text {rad }}\right)(x)=0$.

To complete the proof, it remains to show that $\left\|f-f_{\text {rad }}\right\|_{\infty} \leq C\|\nabla f\|_{N, 1}$ for some constant $C>0$. Recall that (3.11) holds for every $\rho>R$ with $C$ independent of $R$ and $\rho$ (and $f$ ). Thus, $\left\|f-f_{\text {rad }}\right\|_{\infty, \Omega_{\rho}} \leq C\left\|\nabla\left(f-f_{\text {rad }}\right)\right\|_{N, 1} \leq 2 C\|\nabla f\|_{N, 1}$ for every $\rho>0$. Since $\left\|f-f_{\text {rad }}\right\|_{\infty}=\sup _{\rho>0}\left\|f-f_{\text {rad }}\right\|_{\infty, \Omega_{\rho}}$, it follows that $\left\|f-f_{\text {rad }}\right\|_{\infty} \leq$ $C\|\nabla f\|_{N, 1}$ after changing $2 C$ into $C$.

Theorem 3.7 is a special case of a more general property (Theorem 3.10). To prove it, we need

Lemma 3.8. If $\nabla h \in\left(L^{q, 1}\right)^{N}$ with $1 \leq q<N$, there is a unique constant $c_{h} \in \mathbb{R}$ such that $h-c_{h} \in L^{q^{*}, 1}$ and there is a constant $C>0$ independent of $h$ such that $\left\|h-c_{h}\right\|_{q^{*}, 1} \leq C\|\nabla h\|_{q, 1}$.

Proof. With $\Omega=B_{1}$ in Lemma 3.5 (ii) and by an elementary scaling argument, $\left\|h-\bar{h}_{r}\right\|_{q^{*}, 1, B_{r}} \leq C\|\nabla h\|_{q, 1, B_{r}} \leq C\|\nabla h\|_{q, 1}$ for every $r>0$, where $\bar{h}_{r}:=\left|B_{r}\right|^{-1} \int_{B_{r}} h$ and where $C>0$ is independent of $r$ (and $h$ ). Let $\left(r_{n}\right)$ be a sequence tending to $\infty$. Since $\left\|h-\bar{h}_{r_{n}}\right\|_{q^{*}, 1, B_{1}} \leq\left\|h-\bar{h}_{r_{n}}\right\|_{q^{*}, 1, B_{r_{n}}}$ for $n$ large enough, this shows that 
the sequence $\left(\bar{h}_{r_{n}}\right)$ is bounded. After passing to a subsequence, assume with no loss of generality that $\lim \bar{h}_{r_{n}}=c_{h} \in \mathbb{R}$. Since $\left\|h-\bar{h}_{r_{n}}\right\|_{q^{*}, 1, B_{r_{n}}}=\left\|\left(h-\bar{h}_{r_{n}}\right) \chi_{B_{r_{n}}}\right\|_{q^{*}, 1}$ and since $\left(h-\bar{h}_{r_{n}}\right) \chi_{B_{r_{n}}} \rightarrow h-c_{h}$ a.e., it follows from the general form of Fatou's lemma $\left(\left[2\right.\right.$, p. 5]) that $\left\|h-c_{h}\right\|_{q^{*}, 1} \leq \liminf \left\|h-\bar{h}_{r_{n}}\right\|_{q^{*}, 1, B_{r_{n}}} \leq C\|\nabla h\|_{q, 1}$. Since $L^{q^{*}, 1}$ contains no nonzero constant, $c_{h}$ is unique.

Remark 3.9. A mild elaboration on the above proof shows that, by the uniqueness of $c_{h}$, every convergent subsequence of $\left(\bar{h}_{r_{n}}\right)$ converges to $c_{h}$. Thus, $\lim \bar{h}_{r_{n}}=c_{h}$ irrespective of $\left(r_{n}\right)$ and so $c_{h}=\lim _{r \rightarrow \infty}\left|B_{r}\right|^{-1} \int_{B_{r}} h$, as in Remark 3.2 when $c_{h}$ is the radial limit of $h$.

With $L^{q, 1}$ and $L^{q^{*}, 1}$ replaced with $L^{q}$ and $L^{q^{*}}$, respectively, Lemma 3.8 is due to Lizorkin [14], with a different proof (see also [9, Theorem 5.1, p. 64]). Another variant will be proved in Lemma 4.4 .

Theorem 3.10. If $N>1$ and if $\nabla^{k} f \in\left(L^{N / k, 1}\right)^{N^{k}}$ for some $1 \leq k \leq N$ (in particular, if $\left.\nabla^{N} f \in\left(L^{1}\right)^{N^{N}}\right)$, there is a unique polynomial $P_{f}$ of degree at most $k-1$ such that $f-P_{f} \in \mathcal{B U C}$ and that $\lim _{|x| \rightarrow \infty}\left(f(x)-P_{f}(x)\right)=0$. Furthermore, there is a constant $C>0$ independent of $f$ such that $\left\|f-P_{f}\right\|_{\infty} \leq C\left\|\nabla^{k} f\right\|_{N / k, 1}$.

Proof. If $k>1$, it follows from Lemma 3.8 with $h$ any partial derivative of $f$ of order $k$ that there is a unique symmetric covariant tensor $M_{k-1}$ of order $k-1$ such that $\nabla^{k-1} f-M_{k-1} \in\left(L^{(N / k)^{*}, 1}\right)^{N^{k-1}}=\left(L^{N /(k-1), 1}\right)^{N^{k-1}}$ and that $\| \nabla^{k-1} f-$ $M_{k-1}\left\|_{N /(k-1), 1} \leq C\right\| \nabla^{k} f \|_{N / k, 1}$. Since $M_{k-1}=\nabla^{k-1} H_{k-1}$ for a unique homogeneous polynomial $H_{k-1}$ of degree $k-1$, this reads $\nabla^{k-1}\left(f-H_{k-1}\right) \in\left(L^{N /(k-1), 1}\right)^{N^{k-1}}$ and $\left\|\nabla^{k-1}\left(f-H_{k-1}\right)\right\|_{N /(k-1), 1} \leq C\left\|\nabla^{k} f\right\|_{N / k, 1}$. Thus, by repeating the same argument, we find a polynomial $P_{k-1}$ of degree at most $k-1$ such that $\nabla\left(f-P_{k-1}\right) \in\left(L^{N, 1}\right)^{N}$ and $\left\|\nabla\left(f-P_{k-1}\right)\right\|_{N, 1} \leq C\left\|\nabla^{k} f\right\|_{N / k, 1}$ with another constant $C$. This remains trivially true, with $P_{0}=0$, if $k=1$.

By Theorem 1.1 (ii) and Theorem 3.7, $f-P_{k-1} \in \mathcal{B U C}$ and $f-P_{k-1}$ has a uniform finite constant limit $c_{f-P_{k-1}}$ at infinity and $\left\|f-P_{k-1}-c_{f-P_{k-1}}\right\|_{\infty} \leq C \| \nabla(f-$ $\left.P_{k-1}\right) \|_{N, 1}$. Since $\left\|\nabla\left(f-P_{k-1}\right)\right\|_{N, 1} \leq C\left\|\nabla^{k} f\right\|_{N / k, 1}$ (with another constant $C$ ), the result follows with $P_{f}=P_{k-1}-c_{f-P_{k-1}}$. The uniqueness of $P_{f}$ is obvious.

If $H_{i}$ is the homogeneous part of $P_{f}$ of degree $0 \leq i \leq k-1$ and $H_{k} \equiv 0$, it follows from Remark 3.2 and Remark 3.9 that the coefficients of $H_{i}$ are the limits of the averages of the partial derivatives of $f-\left(H_{k}+\ldots+H_{i+1}\right)$ of order $i$ over balls of radius tending to $\infty$.

Theorem 3.10 is a generalization of the well-known embedding $W^{N, 1} \hookrightarrow \mathcal{B U C}$. Indeed, if $\nabla^{k} f \in\left(L^{N / k, 1}\right)^{N^{k}}$ with $1 \leq k \leq N$, any condition on $f$ ensuring that $P_{f}=0$ yields that $f$ is uniformly continuous and tends uniformly to 0 at infinity. For 
instance, $P_{f}=0$ if $f$ vanishes at infinity as defined in the Introduction (in particular, if $f \in L^{p, \sigma}$ with $1 \leq p<\infty$ and $\sigma=1$ if $p=1$ ).

When $k<N$, Mizuta [16, Theorem 3 (ii)] has obtained a result weaker than Theorem 3.10 under the weaker assumption $\nabla^{k} f \in\left(L^{N / k}\right)^{N^{k}}$. His method yields an integral formula for $f-P_{f}$ but $L^{1}$ is excluded, so that $k=N$ is ruled out. Also, under the additional assumption that $f$ vanishes at infinity, Theorem 3.10 with $k=1$ and $P_{f}=0$ follows from Tartar [28, Theorem 8 and Remark 9], where it is only assumed that $1 \leq p_{j}<\infty$ and $\sum_{j=1}^{N} p_{j}^{-1}=1$ (instead of just $p_{j}=N$ ). We do not know whether Theorem 3.10 with $k=1$ is still true in this case. See, however, Theorem 4.2 and Theorem 4.5 for closely related results. See also Theorem 4.9 for a non-isotropic variant of Theorem 3.10 when $k=2$.

\section{The case $\partial_{j} f \in L^{p_{j}, \sigma_{j}}$ with $1 \leq p_{j} \leq N$}

If $\partial_{j} f \in L^{p_{j}, \sigma_{j}}$ with $1 \leq p_{j} \leq N$ and $\sigma_{j}=1$ when $p_{j}=1$, then $f$ need not be bounded or continuous, but of course $f$ is continuous if, in addition, $\partial_{j} f \in L_{l o c}^{N, 1}$. In this section, we show that if this local integrability condition is replaced with a suitable uniformly local one and if $\sigma_{j}=1$ when $p_{j}=N$, the boundedness of $f$, its uniform continuity and the existence of a limit at infinity are recovered as well (Theorem 4.2 and Theorem 4.5). More generally, this can be obtained under UL integrability conditions on the higher order derivatives of $f$ (Theorem 4.7).

Recall the definition of the uniformly local Lorentz space (see the Introduction)

$$
L_{u l o c}^{p, \sigma}:=\left\{h \in L_{l o c}^{p, \sigma}: \sup _{z \in \mathbb{R}^{N}}\|h\|_{p, \sigma, B_{1}(z)}<\infty\right\},
$$

where $B_{1}(z)$ is the unit ball with center $z$, with (quasi-) norm $\|h\|_{p, \sigma, u l o c}:=$ $\sup _{z \in \mathbb{R}^{N}}\|h\|_{p, \sigma, B_{1}(z)}$, where $\|\cdot\|_{p, \sigma, B_{1}(z)}$ is the (quasi-) norm of $L^{p, \sigma}\left(B_{1}(z)\right)$. The definition of $L_{u l o c}^{p, \sigma}$ is unchanged if $B_{1}(z)$ is replaced with $B_{r}(z)$ for some fixed $r>0$ and $L_{u l o c}^{p}=L_{u l o c}^{p, p}$. We shall always assume $1 \leq p, \sigma \leq \infty$ and $\sigma=p$ if $p=1$ or $\infty$, with primary focus on $p=N, \sigma=1$ or $\sigma=p$, but other choices will also be relevant.

If $p=\sigma=\infty$, then $L_{u l o c}^{\infty, \infty}=L_{u l o c}^{\infty}=L^{\infty}$ but $L_{u l o c}^{p, \sigma}$ is larger than $L^{p, \sigma}$ when $p<\infty$. Also, it is readily checked that $L^{\infty} \subset L_{u l o c}^{p_{2}, \sigma_{2}} \subset L_{u l o c}^{p_{1}, \sigma_{1}}$ if $p_{2}>p_{1}$ or if $p_{2}=p_{1}$ and $\sigma_{2} \leq \sigma_{1}$ (just like the usual spaces on bounded domains). This turns out to be an important property. In particular, $L_{u l o c}^{p, \sigma} \subset L_{u l o c}^{1}$. Based on the loose principle that a local condition cannot imply a global one, it is intuitively obvious that if $h \in L^{p, \sigma} \cap L_{u l o c}^{q, \tau}$ and $q<\infty$, then $h$ need not belong to $L^{r, \mu}$ if $r \neq p$ or $r=p$ and $\mu<\sigma$. It is only mildly technical to support this statement by explicit examples.

Lemma 4.1. (i) If $h \in L^{p, \sigma}$ with $1 \leq p \leq N$ and $\sigma=1$ when $p=1$ or $N$, then $|x|^{1-N} * h \in L^{\infty}$ if and only if $|x|^{1-N} \chi_{B_{1}} * h \in L^{\infty}$. If so, there is a constant $C>0$ 
independent of $h$ such that

$$
\left\||x|^{1-N} * h\right\|_{\infty} \leq\left\||x|^{1-N} \chi_{B_{1}} * h\right\|_{\infty}+C\|h\|_{p, \sigma} .
$$

(ii) If $h \in L^{p, \sigma} \cap L_{\text {uloc }}^{N, 1}$ with $1 \leq p \leq N$ and $\sigma=1$ when $p=1$ or $N$, then $|x|^{1-N} * h \in L^{\infty}$. Furthermore, there is a constant $C>0$ such that

$$
\left\||x|^{1-N} * h\right\|_{\infty} \leq C\left(\|h\|_{p, \sigma}+\|h\|_{N, 1, u l o c}\right) .
$$

Proof. (i) The claim is that $|x|^{1-N} \chi_{\mathbb{R}^{N} \backslash B_{1}} * h \in L^{\infty}$. With no loss of generality, assume $h \geq 0$. Observe that $|x|^{1-N} \chi_{\mathbb{R}^{N} \backslash B_{1}} \in L^{p^{\prime}, \sigma^{\prime}}$ since $1 \leq p \leq N$ and $\sigma=1$ when $p=1$ or $N$ and that $\|h(z-\cdot)\|_{p, \sigma}=\|h\|_{p, \sigma}$ for every $z \in \mathbb{R}^{N}$. Thus, by Hölder's inequality, $\left(|x|^{1-N} \chi_{\mathbb{R}^{N} \backslash B_{1}} * h\right)(z) \leq\left\||x|^{1-N} \chi_{\mathbb{R}^{N} \backslash B_{1}}\right\|_{p^{\prime}, \sigma^{\prime}}\|h\|_{p, \sigma}$ and (4.1) follows with $C=\left\||x|^{1-N} \chi_{\mathbb{R}^{N} \backslash B_{1}}\right\|_{p^{\prime}, \sigma^{\prime}}$.

(ii) Once again, assume $h \geq 0$ with no loss of generality. By (i), it suffices to show that $|x|^{1-N} \chi_{B_{1}} * h \in L^{\infty}$. Since $|x|^{1-N} \in L^{N /(N-1), \infty}\left(B_{1}\right)$ and since $L^{N, 1}\left(B_{1}\right)$ is the associate space of $L^{N /(N-1), \infty}\left(B_{1}\right)$, it follows that

$$
\begin{aligned}
& \left(|x|^{1-N} \chi_{B_{1}} * h\right)(z) \leq\left\||x|^{1-N}\right\|_{N /(N-1), \infty, B_{1}}\|h(z-\cdot)\|_{N, 1, B_{1}} \\
& \quad=\left\||x|^{1-N}\right\|_{N /(N-1), \infty, B_{1}}\|h\|_{N, 1, B_{1}(z)} \leq\left\||x|^{1-N}\right\|_{N /(N-1), \infty, B_{1}}\|h\|_{N, 1, u l o c}
\end{aligned}
$$

for every $z \in \mathbb{R}^{N}$. Hence, $|x|^{1-N} \chi_{B_{1}} * h \in L^{\infty}$. To prove (4.2), use (4.1) and (4.3).

The first part of Theorem 4.2 below is a strengthening of the Lusin property. A smooth function $f$ coinciding with $\log \log |x|$ for large $|x|$ is a counter-example when $p_{j} \geq N>1$.

Theorem 4.2. Assume $N>1$.

(i) If $\partial_{j} f \in L^{p_{j}, \sigma_{j}}$ with $1 \leq p_{j} \leq N$ and $\sigma_{j}=1$ if $p_{j}=1$ or $N$, there are a subset $E \subset \mathbb{R}^{N}$ of arbitrarily small measure and a function $f_{E} \in \mathcal{C}_{b}$ such that $f(x)=f_{E}(x)$ for $x \notin E$. (ii) If $\partial_{j} f \in L^{p_{j}, \sigma_{j}} \cap L_{u l o c}^{N, 1}$ with $1 \leq p_{j} \leq N$ and $\sigma_{j}=1$ if $p_{j}=1$ or $N$, then $f \in \mathcal{C}_{b}$ and Theorem 3.1 ( $i$ ) is applicable. Furthermore, there is a constant $C>0$ independent of $f$ such that $\|f\|_{\infty} \leq \inf |f|+C \sum_{j=1}^{N}\left(\left\|\partial_{j} f\right\|_{p_{j}, \sigma_{j}}+\left\|\partial_{j} f\right\|_{N, 1, u l o c}\right)$.

(iii) If, in (ii), $\lim _{\rho \rightarrow 0} \sup _{z \in \mathbb{R}^{N}}\left\|\partial_{j} f\right\|_{N, 1, B_{\rho}(z)}=0$ for every $j$, then $f \in \mathcal{B U C}$ and Theorem 3.1 (ii) is applicable. In particular, this holds if $\partial_{j} f \in L^{p_{j}, \sigma_{j}} \cap L_{\text {uloc }}^{q}$ with $q>N$ for every $j$ such that $p_{j}<N$.

Proof. (i) The Riesz potential $I_{1}$ is bounded from $L^{1}$ into $L^{N /(N-1), \infty}$, from $L^{N, 1}$ into $L^{\infty}$ and from $L^{p_{j}, \sigma_{j}}$ into $L^{p_{j}^{*}, \sigma_{j}}$ if $1<p_{j}<N([2$, Theorem 4.18, p. 228]). Thus, irrespective of $j$, the set $F_{j, a}:=\left\{z \in \mathbb{R}^{N}:\left(|x|^{1-N} *\left|\partial_{j} f\right|\right)(z)>a\right\}$ has arbitrarily small measure if $a>0$ is large enough and so the set $F_{a}:=\left\{z \in \mathbb{R}^{N}:\left(|x|^{1-N_{*}}\right.\right.$ $|\nabla f|)(z)>a\} \subset \cup_{j=1}^{N} F_{j, a / \sqrt{N}}$ has arbitrarily small measure if $a>0$ is large enough. By 
Theorem 2.3 and Lemma 2.4 with $S=\mathbb{R}^{N} \backslash F_{a}, \rho_{S}=\infty$ and $C_{S}=a\left|\mathbb{S}^{N-1}\right|^{-1}$, it follows that $|f(y)-f(z)|$ is uniformly bounded when $y, z \in \mathbb{R}^{N} \backslash F_{a}$ and so $f \in L^{\infty}\left(\mathbb{R}^{N} \backslash F_{a}\right)$, say $|f(x)| \leq M$ with $M<\infty$ when $x \notin F_{a}$. By Lusin's theorem, there are a subset $F \subset \mathbb{R}^{N}$ with arbitrarily small measure and a continuous function $f_{F}$ such that $f(x)=f_{F}(x)$ when $x \notin F$. If $E:=F_{a} \cup F$ and $f_{E}:=\max \left\{-M, \min \left\{f_{F}, M\right\}\right\}$, then $f_{E} \in$ $\mathcal{C}_{b}$ and $f(x)=f_{E}(x)$ if $x \notin E$, a set of arbitrary small measure. This proves (i).

(ii) Use Lemma 4.1 (ii) with $h=\left|\partial_{j} f\right|$ and Theorem 1.1 (i) for the boundedness and [26] for the continuity.

(iii) For the uniform continuity, see Remark 2.5. If $p_{j}=N$, then $\partial_{j} f \in L^{N, 1}$ and $\lim _{\rho \rightarrow 0} \sup _{z \in \mathbb{R}^{N}}\left\|\partial_{j} f\right\|_{N, 1, B_{\rho}(z)}=0$ holds by the absolute continuity of the $L^{N, 1}$ norm, as in the proof of Theorem 1.1 (ii). Suppose now that $\partial_{j} f \in L^{p_{j}, \sigma_{j}} \cap L_{u l o c}^{q}$ with $q>N$. Since $L^{q}\left(B_{1}\right) \hookrightarrow L^{N, 1}\left(B_{1}\right)$, there is a constant $C>0$ such that $\|h\|_{N, 1, B_{1}} \leq$ $C\|h\|_{q, B_{1}}$ for every $h \in L^{q}\left(B_{1}\right)$ and, by scaling, $\|h\|_{N, 1, B_{\rho}} \leq C \rho^{1-N / q}\|h\|_{q, B_{\rho}}$ with the same constant $C$ for every $\rho>0$ and every $h \in L^{q}\left(B_{\rho}\right)$. By translation invariance, $\|h\|_{N, 1, B_{\rho}(z)} \leq C \rho^{1-N / q}\|h\|_{q, B_{\rho}(z)}$ for every $z \in \mathbb{R}^{N}$, every $\rho>0$ and every $h \in$ $L^{q}\left(B_{\rho}(z)\right)$.

With $h=\partial_{j} f$, we get $\left\|\partial_{j} f\right\|_{N, 1, B_{\rho}(z)} \leq C \rho^{1-N / q}\left\|\partial_{j} f\right\|_{q, u l o c}$ for every $z \in \mathbb{R}^{N}$ and every $\rho \leq 1$ and so $\lim _{\rho \rightarrow 0} \sup _{z \in \mathbb{R}^{N}}\left\|\partial_{j} f\right\|_{N, 1, B_{\rho}(z)}=0$.

It is known (see [28, Remark 9]) that $f \in \mathcal{B U C}$ if $f$ vanishes at infinity and $\sum_{j=1}^{N} p_{j}^{-1}<1$. In contrast, in Theorem 4.2, there is no assumption on $f$ itself and $\sum_{j=1}^{N} p_{j}^{-1} \geq 1$ (but $\nabla f \in\left(L_{u l o c}^{N, 1}\right)^{N}$ ). We do not know whether Theorem 4.2 remains true if $\sum_{j=1}^{N} p_{j}^{-1} \geq 1$ is assumed instead of just $p_{j} \leq N$, but without the assumption that $f$ vanishes at infinity, it is false if $\sum_{j=1}^{N} p_{j}^{-1}<1$. For example, if $\sigma_{j}=p_{j}=p>N$, then $\partial_{j} f \in L^{p} \cap L_{\text {uloc }}^{N, 1}$ (because $L^{p} \subset L_{u l o c}^{p} \subset L_{u l o c}^{N, 1}$ ) and $f$ is uniformly continuous (by Morrey's theorem) but generally unbounded (example: $f(x)=(1+|x|)^{\varepsilon}$ with $0<\varepsilon<$ $1-N / p)$.

When $p_{j}=p<N<q$ and $\partial_{j} f \in L^{p} \cap L^{q}$ (but not $L^{p} \cap L_{u l o c}^{q}$ ), Theorem 4.2 (iii) and Theorem 4.5 below follow from Galdi [9].

Example 4.3. Suppose that $\nabla f \in\left(L^{p}\right)^{N}$ with $1 \leq p<N$ and that $|\nabla f| \leq h$ with $h(x):=C\left(1+|x|^{-\beta}\right)$, where $C>0$ and $0<\beta<1$. Then, $h \notin L^{q}$ for any $1 \leq q \leq \infty$, but since $|x|^{-\beta}$ is bounded outside $B_{1}$ and $|x|^{-\beta} \in L^{q}\left(B_{1}\right)$ for some $q>N$ (use $\beta<1$ ), it follows that $h \in L^{\infty}+L^{q} \subset L_{u l o c}^{q}$. Hence, Theorem 4.2 (iii) applies.

One may argue that, in this example, Theorem 4.2 allows for a quick proof but that it is not indispensable. Indeed, $h \in L^{\infty}+L^{q}$ implies $\nabla f \in\left(L^{\infty}+L^{q}\right)^{N}$, whence $\nabla f \in\left(L^{p} \cap\left(L^{\infty}+L^{q}\right)\right)^{N}$. Now, it is not hard to check that $p<q<\infty$ implies $L^{p} \cap$ $\left(L^{\infty}+L^{q}\right)=L^{p} \cap L^{q}$ (obviously, this is not an algebraic property). Consequently, 
$\nabla f \in\left(L^{p} \cap L^{q}\right)^{N} \subset\left(L^{N, 1}\right)^{N}$ since $p<N<q$ and the result follows from an already known special case of Theorem 1.1 (ii).

Far from downplaying the value of the UL spaces, this reveals that their use may make it possible to bypass technical properties, possibly much harder to notice than $L^{p} \cap\left(L^{\infty}+L^{q}\right)=L^{p} \cap L^{q}$, if they exist at all. This greatly simplifying feature is directly related to the fact that the UL spaces are linearly ordered by inclusion. See the comments after Corollary 4.8 for corroborative evidence.

Naturally, there are instances when there is clearly no substitute for Theorem 4.2. For instance, when $h$ in Example 4.3 is changed into a nonzero $N$-periodic $h \in L_{l o c}^{N, 1}$ or $h \in L_{l o c}^{q}$ with $N<q<\infty$ (so that $h \in L_{u l o c}^{N, 1}$ or $L_{u l o c}^{q}$ by periodicity).

We now show that depending upon the distribution of the $p_{j}$ in Theorem 4.2 (iii), the constant limit of $f$ at infinity is uniform. Part (iii) of the next lemma is the important ingredient but the other parts are needed for its proof.

Lemma 4.4. For $j=1, \ldots, N$ with $N>1$, let $1 \leq p_{j}<\infty$ and $1 \leq \sigma_{j} \leq \infty$ be such that $\sum_{j=1}^{N} p_{j}^{-1}>1$ and that $\sigma_{j}=1$ if $p_{j}=1$. Set $p_{\min }:=\min _{j} p_{j}<N, p_{\max }:=$ $\max _{j} p_{j}<\infty$ and

$$
p^{\sharp}:=N\left(\sum_{j=1}^{N} p_{j}^{-1}-1\right)^{-1}>p_{\min }, \quad \sigma:=N\left(\sum_{j=1}^{N} \sigma_{j}^{-1}\right)^{-1} .
$$

Suppose further that $p_{\max }<p^{\sharp}$ and let $Q$ denote an open rectangle with edges parallel to the coordinate axes. Then, the following properties hold:

(i) If $h \in \mathcal{D}^{\prime}(Q)$ and $\partial_{j} h \in L^{p_{j}, \sigma_{j}}(Q)$, then $h \in L^{p^{\sharp}, \sigma}(Q)$.

(ii) (Poincaré-Wirtinger inequality) There is a constant $C>0$ such that $\|h\|_{p^{\sharp}, \sigma, Q} \leq$ $C \sum_{j=1}^{N}\left\|\partial_{j} h\right\|_{p_{j}, \sigma_{j}, Q}$ for every $h \in \mathcal{D}^{\prime}(Q)$ such that $\partial_{j} h \in L^{p_{j}, \sigma_{j}}(Q)$ and $\int_{Q} h=0$ (unambiguous since $h \in L^{1}(Q)$ by $\left.(i)\right)$.

(iii) If $\partial_{j} f \in L^{p_{j}, \sigma_{j}}$, there is a unique constant $c_{f} \in \mathbb{R}$ such that $f-c_{f} \in L^{p^{\sharp}, \sigma}$.

Proof. (i) If $\sigma_{j}=p_{j}$, i.e., $\partial_{j} h \in L^{p_{j}}(Q)$, then $\nabla h \in\left(L^{p_{\min }}(Q)\right)^{N}$ and, hence, $h \in$ $L^{p_{\min }}(Q)$. By Rákosník [23] (see also [10]), $h \in L^{p^{\sharp}}(Q)$ (this does not use $p_{\max }<p^{\sharp}$ ).

In general, if $\partial_{j} h \in L^{p_{j}, \sigma_{j}}(Q)$, then $\partial_{j} h \in L^{q_{j}}(Q)$ with $q_{j}=1$ if $p_{j}=1$ and $1 \leq q_{j}<$ $p_{j}$ otherwise. If $q_{j}$ is close enough to $p_{j}$ for every $j$, then $\sum_{j=1}^{N} q_{j}^{-1}>1$ and so, from the above, $h \in L^{q^{\sharp}}(Q)$ with $q^{\sharp}$ is arbitrarily close to $p^{\sharp}$. Hence, $h \in L^{q}(Q)$ for every $1 \leq q<p^{\sharp}$. In particular, $h \in L^{p_{\max }}(Q)$.

The standard reflection procedure produces a rectangle $\widetilde{Q}$ with edges parallel to the coordinate axes such that $Q \Subset \widetilde{Q}$, along with an extension $\tilde{h} \in L^{p_{\max }}(\widetilde{Q})$ of $h$ such that $\partial_{j} \tilde{h} \in L^{p_{j}, \sigma_{j}}(\widetilde{Q})$. Now, let $\varphi \in \mathcal{C}_{0}^{\infty}$ be such that $\operatorname{Supp} \varphi \subset \widetilde{Q}$ and $\varphi=1$ on $Q$. Then, $\partial_{j}(\varphi \tilde{h})=\left(\partial_{j} \varphi\right) \tilde{h}+\varphi\left(\partial_{j} \tilde{h}\right) \in L^{p_{j}, \sigma_{j}}$ since $L^{p_{\max }} \subset L^{p_{j}, \sigma_{j}}$. Also, $\varphi \tilde{h} \in L^{p_{\max }}$ vanishes at infinity. By [28, Theorem 8], $\varphi \tilde{h} \in L^{p^{\sharp}, \sigma}$ and so $h \in L^{p^{\sharp}}, \sigma(Q)$. 
(ii) The space $W(Q):=\left\{h \in L^{1}(Q): \partial_{j} h \in L^{p_{j}, \sigma_{j}}(Q)\right\}$ is a Banach space for the obvious norm and, by (i), $W(Q) \subset L^{p^{\sharp}, \sigma}(Q)$. The closed graph theorem shows at once that this embedding is continuous, for if $h_{n} \in W(Q)$ tends to $h$ in $W(Q)$ and tends to $g$ in $L^{p^{\sharp}, \sigma}(Q)$, then $h_{n}$ tends to both $h$ and $g$ in $L^{1}(Q)$ and so $h=g$. On the other hand, it is plain that $W(Q) \hookrightarrow W^{1,1}(Q)$ and, since the embedding $W^{1,1}(Q) \hookrightarrow L^{1}(Q)$ is compact, the embedding $W(Q) \hookrightarrow L^{1}(Q)$ is compact.

By the usual contradiction argument, it follows that $\sum_{j=1}^{N}\left\|\partial_{j} h\right\|_{p_{j}, \sigma_{j}, Q}$ is a norm equivalent to the $W(Q)$ norm of $h$ on the subspace $W_{0}(Q)$ of functions with zero average. Hence, the continuity of the embedding $W_{0}(Q) \hookrightarrow L^{p^{\sharp}, \sigma}(Q)$ is accounted for by the inequality $\|h\|_{p^{\sharp}, \sigma, Q} \leq C \sum_{j=1}^{N}\left\|\partial_{j} h\right\|_{p_{j}, \sigma_{j}, Q}$ for every $h \in W_{0}$.

(iii) Set $\theta_{j}:=1 / p_{j}-1 / p^{\sharp}$ and, for $\rho>0$, let $Q_{\rho}:=\prod_{j=1}^{N}\left(-\rho^{\theta_{j}}, \rho^{\theta_{j}}\right)$. For future use, note that the assumption $p_{\max }<p^{\sharp}$. is equivalent to $\theta_{j}>0$ for every $j$. We claim that there is a constant $C>0$ independent of $\rho$ such that

$$
\|h\|_{p^{\sharp}, \sigma, Q_{\rho}} \leq C \sum_{j=1}^{N}\left\|\partial_{j} h\right\|_{p_{j}, \sigma_{j}, Q_{\rho}},
$$

whenever $\partial_{j} h \in L^{p_{j}, \sigma_{j}}\left(Q_{\rho}\right)$ and $\int_{Q_{\rho}} h=0$.

By (ii), $C$ exists when $\rho=1$. In general, set $h_{\rho}(x):=h\left(\rho^{\theta_{1}} x_{1}, \ldots, \rho^{\theta_{N}} x_{N}\right)$ for $x \in Q_{1}$, so that $\partial_{j} h_{\rho} \in L^{p_{j}, \sigma_{j}}\left(Q_{1}\right)$ and $\int_{Q_{1}} h_{\rho}=0$. Then, with $h_{\rho}$ substituted for $h$ in (4.5) with $\rho=1$, the left-hand side is $\rho^{-1 / p^{\sharp}}\|h\|_{p^{\sharp}, \sigma, Q_{\rho}}$ and the right-hand side is $C \sum_{j=1}^{N} \rho^{\theta_{j}-1 / p_{j}}\left\|\partial_{j} h\right\|_{p_{j}, \sigma_{j}, Q_{\rho}}$ (use $\sum_{j=1}^{N} \theta_{j}=1$ ). From the definitions of $\theta_{j}$ and $p^{\sharp}$, it follows that the powers of $\rho$ cancel out and (4.5) holds with the same $C$ as when $\rho=1$.

Now, if $\partial_{j} f \in L^{p_{j}, \sigma_{j}}$, then $\partial_{j} f \in L^{p_{j}, \sigma_{j}}\left(Q_{n}\right)$ for every $n \in \mathbb{N}$. With $\rho=n, h=f-\bar{f}_{n}$ and $\bar{f}_{n}:=\left|Q_{n}\right|^{-1} \int_{Q_{n}} f,(4.5)$ reads

$$
\left\|f-\bar{f}_{n}\right\|_{p^{\sharp}, \sigma, Q_{n}} \leq C \sum_{j=1}^{N}\left\|\partial_{j} f\right\|_{p_{j}, \sigma_{j}, Q_{n}} \leq C \sum_{j=1}^{N}\left\|\partial_{j} f\right\|_{p_{j}, \sigma_{j}} .
$$

As noted earlier, $\theta_{j}>0$ for every $j$, which in turn ensures that $\left(Q_{n}\right)$ is an increasing sequence of rectangles whose union is $\mathbb{R}^{N}$. In particular, from (4.6), $\| f-$ $\bar{f}_{n} \|_{p^{\sharp}, \sigma, Q_{1}}\left(\leq\left\|f-\bar{f}_{n}\right\|_{p^{\sharp}, \sigma, Q_{n}}\right)$ is bounded, so that $\left(\bar{f}_{n}\right)$ is bounded. After passing to a subsequence, assume $\bar{f}_{n} \rightarrow c_{f} \in \mathbb{R}$ with no loss of generality. Then, $\left(f-\bar{f}_{n}\right) \chi_{Q_{n}} \rightarrow$ $f-c_{f}$ a.e. on $\mathbb{R}^{N}$ and so, by (4.6) and Fatou's lemma, $\left\|f-c_{f}\right\|_{p^{\sharp}, \sigma} \leq$ $C \sum_{j=1}^{N}\left\|\partial_{j} f\right\|_{p_{j}, \sigma_{j}}<\infty$. That $c_{f}$ is unique is obvious.

There are counter-examples to (i) for non-rectangular domains with smooth boundary ([10]). The condition $p_{\max }<p^{\sharp}$ is also equivalent to $\sum_{j=1}^{N} p_{j}^{-1}<1+N p_{\max }^{-1}$. 
It holds if $N=2$ or if $p_{\max }<p_{\min }^{*}$ (e.g., $p_{j}=p$ or $\left.N / 2 \leq p_{j}<N\right)$, among other options (e.g., $\left.p_{\max }<(N-1) /(N-2)\right)$. It is probably not needed in (i) and (ii) but it is crucial to the proof of (iii). If it fails, the sequence $\left(Q_{n}\right)$ does not cover $\mathbb{R}^{N}$ and it is not even increasing if $p_{\max }>p^{\sharp}$.

Theorem 4.5. If, in part (iii) of Theorem $4.2, p_{\max }:=\max p_{j}<p^{\sharp}($ see (4.4)), there is a constant $c_{f} \in \mathbb{R}$ such that $\lim _{|x| \rightarrow \infty} f(x)=c_{f}$.

Proof. If $p_{j}=N$ (hence $\sigma_{j}=1$ ) for every $j$, the result follows from Theorem 3.7 (and $p_{\max }=N<\infty=p^{\sharp}$ ). If $p_{j}<N$ for at least one $j$, then $\sum_{j=1}^{N} p_{j}^{-1}>1$, as required in Lemma 4.4. Since $p_{\max }<p^{\sharp}$, part (iii) of that lemma yields a constant $c_{f} \in \mathbb{R}$ such that $f-c_{f} \in L^{p^{\sharp}}, \sigma$. Since $p^{\sharp}<\infty$, the function $f-c_{f}$ vanishes at infinity. On the other hand, by Theorem 4.2 (iii), $f-c_{f} \in \mathcal{B U C}$ and a uniformly continuous function vanishing at infinity tends uniformly to 0 at infinity.

The proof when $p_{j}<N$ for at least one $j$ does not work when $p_{j}=N$ for every $j$ (Theorem 3.7) and vice-versa. In other words, Theorem 3.7 and Theorem 4.5 cannot be proved in one stroke.

Our next goal is to generalize parts (ii) and (iii) of Theorem 4.2 as well as Theorem 4.5 when UL integrability conditions are placed on the higher derivatives of $f$ instead of $\nabla f$. This will be done in the simplest way possible, by showing that such conditions exist that imply that Theorem 4.2 is applicable. We need the following lemma.

Lemma 4.6. (i) If $h \in L_{\text {uloc }}^{1}$ and $\nabla h \in\left(L_{\text {uloc }}^{q}\right)^{N}$ with $1 \leq q<N$, then $h \in L_{\text {uloc }}^{q^{*}}$. (ii) If $h \in L_{\text {uloc }}^{1}$ and $\nabla h \in\left(L_{\text {uloc }}^{q, 1}\right)^{N}$ with $1 \leq q<N$, then $h \in L_{u l o c}^{q^{*}, 1}$.

Proof. (i) Since $\nabla h \in\left(L_{u l o c}^{q}\right)^{N}$, it follows that $\nabla h \in\left(L^{q}\left(B_{1}(z)\right)\right)^{N}$ and, hence, that $h \in W^{1, q}\left(B_{1}(z)\right)$ for every $z \in \mathbb{R}^{N}$. By the classical Poincaré-Wirtinger inequality, $\|h-\bar{h}\|_{q^{*}, B_{1}(z)} \leq C\|\nabla h\|_{q, B_{1}(z)}$ where $\bar{h}:=\left|B_{1}\right|^{-1} \int_{B_{1}(z)} h$ and $C>0$ is a constant independent of $h$ and $z$, the latter by the translation invariance of the Lebesgue measure. As a result, $\|h\|_{q^{*}, B_{1}(z)} \leq\left|B_{1}\right|^{-\left(1 / N+1 / q^{\prime}\right)}\|h\|_{1, B_{1}(z)}+C\|\nabla h\|_{q, B_{1}(z)}$. By taking the supremum over $z \in \mathbb{R}^{N}$, it follows that $h \in L_{u l o c}^{q^{*}}$ with $\|h\|_{q^{*}, u l o c} \leq$ $\left|B_{1}\right|^{-\left(1 / N+1 / q^{\prime}\right)}\|h\|_{1, u l o c}+C\|\nabla h\|_{q, u l o c}$.

(ii) The proof proceeds as above, starting with the Poincaré-Wirtinger inequality $\|h-\bar{h}\|_{q^{*}, 1, B_{1}(z)} \leq C\|\nabla h\|_{q, 1, B_{1}(z)}$ of Lemma 3.5 (ii) (once again, $C$ is independent of $z$ by translation invariance).

The example when $h$ is a nonconstant affine polynomial shows that the assumption $h \in L_{\text {uloc }}^{1}$ cannot be dropped. 
Theorem 4.7. Assume $N>1, \partial_{j} f \in L^{p_{j}, \sigma_{j}}$ with $1 \leq p_{j} \leq N$ and $\sigma_{j}=1$ if $p_{j}=1$ or $N$ and, for some integer $1 \leq k \leq N$ :

(i) $\nabla^{i} f \in\left(L_{\text {uloc }}^{1}\right)^{N^{i}}$ if $1 \leq i \leq k-1$ (always true when $i=1$ ),

(ii) $\nabla^{k} f \in\left(L_{u l o c}^{N / k}\right)^{N^{k}}$.

Then, $f \in \mathcal{C}_{b}$ and Theorem $3.1(i)$ is applicable. Furthermore, if $\nabla^{k} f \in\left(L_{\text {uloc }}^{q_{k}}\right)^{N^{k}}$ with $q_{k}>N / k$ (in particular, if $k=N$ and $\left.\nabla^{N+1} f \in\left(L_{u l o c}^{1}\right)^{N^{N+1}}\right)$, then $f \in \mathcal{B U C}$ and Theorem 3.1 (ii) is applicable. If, in addition, $\max _{j} p_{j}<p^{\sharp}($ see $(4.4))$, there is a constant $c_{f} \in \mathbb{R}$ such that $\lim _{|x| \rightarrow \infty} f(x)=c_{f}$.

Proof. If $k=1$, the hypotheses of Theorem 4.2 (ii) are satisfied, as are those of Theorem 4.2 (iii) (with $q=q_{1}$ ) if $\nabla f \in\left(L_{u l o c}^{q_{1}}\right)^{N}$ with $q_{1}>N$. Thus, everything follows from Theorem 4.2 and Theorem 4.5.

If $k>1$, then by (i) and (ii) and Lemma 4.6 (ii) with $1 \leq q=N / k<N$ and $h$ any partial derivative of $f$ of order $k-1$, it follows from the assumption $\nabla^{k} f \in\left(L_{u l o c}^{N / k, 1}\right)^{N^{k}}$ that $\nabla^{k-1} f \in\left(L_{u l o c}^{N /(k-1), 1}\right)^{N^{k-1}}$ (use $\left.(N / k)^{*}=N /(k-1)\right)$. This reduces the problem to the case when $k$ is replaced with $k-1$ and, by induction, to the case $k=1$ already settled.

If $\nabla^{k} f \in\left(L_{u l o c}^{q_{k}}\right)^{N^{k}}$ with $q_{k}>N / k$ (and $k>1$ ), it is not restrictive to assume $q_{k}<N$ and then $\nabla^{k-1} f \in\left(L_{u l o c}^{q_{k}^{*}}\right)^{N^{k-1}}$ by Lemma 4.6 (i). Since $q_{k}^{*}>N /(k-1)$, the problem is once again reduced to the case when $k$ is replaced with $k-1$ (with $\left.q_{k-1}=q_{k}^{*}\right)$ and, hence, by induction, to the case $k=1$ already settled.

In particular, if $k=N$ and $\nabla^{N+1} f \in\left(L_{u l o c}^{1}\right)^{N^{N+1}}$, then $\nabla^{N} f \in\left(L_{u l o c}^{1^{*}}\right)^{N^{N}}$ by Lemma 4.6 (i) and $q_{N}:=1^{*}=N /(N-1)>1=N / N$.

We spell out the special case of Theorem 4.7 in the setting of classical Lebesgue spaces. The proof follows at once from Theorem 4.7 and from $L^{p} \subset L_{u l o c}^{p} \subset L_{u l o c}^{q, 1}$ for every $q<p$. Also, $\gamma:=\left(\gamma_{1}, \ldots, \gamma_{N}\right)$ is a multi-index with nonnegative integer entries and $|\gamma|_{1}:=\gamma_{1}+\ldots+\gamma_{N}$.

Corollary 4.8. Assume $N>1, \partial_{j} f \in L^{p_{j}}$ with $1 \leq p_{j}<N$ and, for some integer $1 \leq k \leq N+1$ :

(i) $\partial^{\gamma} f \in L^{p_{\gamma}}$ for some $1 \leq p_{\gamma} \leq \infty$ when $1 \leq|\gamma|_{1} \leq k-1$ (always true when $|\gamma|_{1}=1$ ),

(ii) $\partial^{\gamma} f \in L^{p_{\gamma}}$ for some $N / k<p_{\gamma} \leq \infty$ when $|\gamma|_{1}=k \leq N$, or $\partial^{\gamma} f \in L^{p_{\gamma}}$ for some $1 \leq$ $p_{\gamma} \leq \infty$ when $|\gamma|_{1}=k=N+1$.

Then, $f \in \mathcal{B U C}$ and Theorem 3.1 (ii) is applicable. If , in addition, $\max _{j} p_{j}<p^{\sharp}$ (see (4.4)), there is a constant $c_{f} \in \mathbb{R}$ such that $\lim _{|x| \rightarrow \infty} f(x)=c_{f}$.

Without drastic limitations, Corollary 4.8 cannot be proved without using the UL spaces. For example, it states that $f \in \mathcal{B U C}$ if $\partial_{j} f \in L^{p_{j}}$ with $1 \leq p_{j}<N$ and $\partial^{\gamma} f \in L^{p_{\gamma}}$ for any $1 \leq p_{\gamma} \leq \infty$ when $2 \leq|\gamma|_{1} \leq N+1$. It should be clear that the known embedding theorems, including anisotropic ones, do not come close to yielding an 
alternate direct proof of just the uniform continuity of $f$ (its continuity follows from $f \in W_{l o c}^{N+1,1}$; the difficulty is with global properties).

There is a variant of Theorem 3.10 when assumptions are made only on the derivatives of $f$ of order $k \geq 2$, at least when $k \leq N-1$, but the general hypotheses are rather convoluted. Accordingly, we just give the statement when $k=2$ (when $k=1$, see Theorem 4.2 (ii) and (iii) and Theorem 4.5).

Theorem 4.9. Assume $N>2, \partial_{i j}^{2} f \in L^{p_{i j}, \sigma_{i j}} \cap L_{u l o c}^{N / 2}$ with $1 \leq p_{i j}=p_{j i}<\infty$ and $\sigma_{i j}=1$ if $p_{i j}=1$ and $\sum_{i=1}^{N} p_{i j}^{-1}>2$ for $1 \leq j \leq N$ (this cannot hold if $\left.N=2\right)$. Set $p_{j}^{\sharp}:=$ $N\left(\sum_{i=1}^{N} p_{i j}^{-1}-1\right)^{-1} \in(1, N)$ and assume further $\max _{i} p_{i j}<p_{j}^{\sharp}$ for $1 \leq j \leq N$. Then:

(i) There is an affine polynomial $P_{f}$ such that $f-P_{f} \in \mathcal{C}_{b}$ and that $\lim _{r \rightarrow \infty} f(z+$ $r \omega)-P_{f}(z+r \omega)=0$ for every $z \in \mathbb{R}^{N}$ and a.e. $\omega \in \mathbb{S}^{N-1}$ (depending upon $z$ ).

(ii) If $\partial_{i j}^{2} f \in L^{p_{i j}, \sigma_{i j}} \cap L_{u l o c}^{q}$ with $q>N / 2$, then $f-P_{f} \in \mathcal{B U C}$ and $\lim _{r \rightarrow \infty} f(z+r \omega)-$ $P_{f}(z+r \omega)=0$ for every $z \in \mathbb{R}^{N}$ and a.e. $\omega \in \mathbb{S}^{N-1}$ (independent of $z$ ).

(iii) If, in (ii), $\max _{j} p_{j}^{\sharp}<p^{\sharp \sharp}:=N\left(\sum_{j=1}^{N} p_{j}^{\sharp-1}-1\right)^{-1}$, then $\lim _{|x| \rightarrow \infty}(f(x)-$ $\left.P_{f}(x)\right)=0$.

Proof. (i) Fix $1 \leq j \leq N$. Then, $\partial_{i}\left(\partial_{j} f\right) \in L^{p_{i j}, \sigma_{i j}}$ for $1 \leq i \leq N$ with $\sum_{i=1}^{N} p_{i j}^{-1}>$ $2>1$ and $\max _{i} p_{i j}<p_{j}^{\sharp}$. Thus, by Lemma 4.4 (iii) with $h=\partial_{j} f$, there is $c_{j} \in \mathbb{R}$ such that $\partial_{j} f-c_{j} \in L^{p_{j}^{\sharp}, \sigma_{j}}$ with $\sigma_{j}:=N\left(\sum_{i=1}^{N} \sigma_{i j}^{-1}\right)^{-1}$.

In particular, $\partial_{j} f-c_{j} \in L_{u l o c}^{1}$ and $\nabla\left(\partial_{j} f-c_{j}\right)=\nabla \partial_{j} f \in\left(L_{u l o c}^{N / 2,1}\right)^{N}$. Since $(N / 2)^{*}=N$, it follows from Lemma 4.6 (ii) that $\partial_{j} f-c_{j} \in L_{u l o c}^{N, 1}$.

In summary, $\partial_{j} f-c_{j} \in L^{p_{j}^{\sharp}, \sigma_{j}} \cap L_{u l o c}^{N, 1}$ with $1<p_{j}^{\sharp}<N$ and $\partial_{j} f-c_{j}=\partial_{j}\left(f-Q_{f}\right)$ where $Q_{f}(x):=\sum_{i=1}^{N} c_{i} x_{i}$. Thus, by Theorem 4.2 (ii), $f-Q_{f} \in \mathcal{C}_{b}$ and Theorem 3.1 (i) is applicable to $f-Q_{f}$. This proves (i) with $P_{f}:=Q_{f}+c$ for some $c \in \mathbb{R}$.

(ii) Since $\partial_{i j}^{2} f \in L_{u l o c}^{q}$ with $q>N / 2$, we may assume with no loss of generality that $q<N$. Then, in the proof of (i) above, Lemma 4.6 (ii) yields $\partial_{j} f-c_{j} \in L_{u l o c}^{q^{*}, 1}$, so that $\partial_{j}\left(f-Q_{f}\right) \in L^{p_{j}^{\sharp}, \sigma_{j}} \cap L_{u l o c}^{q^{*}, 1}$. Since $q^{*}>N$, it follows from Theorem 4.2 (iii) that $f-Q_{f} \in \mathcal{B U C}$ and that Theorem 3.1 (ii) is applicable to $f-Q_{f}$. Hence, $f-P_{f} \in \mathcal{B U C}$ since $P_{f}-Q_{f}$ is constant.

(iii) This follows at once from Theorem 4.5 with $f$ replaced with $f-Q_{f}$.

All the conditions on $p_{i j}$ and $p_{j}^{\sharp}$ in Theorem 4.9 are satisfied when $p_{i j}=p<N / 2$. If so, $p_{j}^{\sharp}=p^{*}=N p /(N-p)$ and $p^{\sharp \sharp}=\left(p^{*}\right)^{*}=N p /(N-2 p)$.

\section{Application to convolution}

Assume $\partial_{j} f \in L^{p_{j}, \sigma_{j}}$ with $1 \leq p_{j} \leq N$ and $\sigma_{j}=1$ if $p_{j}=1$ or $N$ (no UL integrability condition). We now discuss the boundedness and continuity of $g * f$ for suitable $g$. 
The first task is to verify that $g * f$ is defined. Since $f$ need not belong to any $L^{p, \sigma}$, this is not a routine matter unless $g \in L^{1}$ has compact support, a case of limited interest. However, as we shall see, $g * f$ is always defined when $g \in L^{1}$ (or in a more general class of distributions), even though this does not follow from Young's theorem or from its generalization to Lorentz spaces.

Rather unexpectedly, Theorem 1.1 (ii) plays a key role, along with results of Schwartz $([24])$, in the proof that $g * f$ is a well defined distribution when $g \in L^{1}$. It also implies, under additional assumptions on $g$, that $g * f$ is a $\mathcal{B U C}$ function having a uniform finite constant limit at infinity (Theorem 5.2 (iii), Theorem 5.7). The same result can be obtained when $g \in L^{1}$ and $f$ satisfies the conditions of Theorem 4.7 or Corollary 4.8 (Theorem 5.6).

To begin with, we recall some properties of convolution in Lorentz spaces. We only record what is needed later.

Lemma 5.1. (i) Assume $1 \leq p, \sigma \leq \infty$ and $\sigma=p$ if $p=1$ or $\infty$. If $g \in L^{1}$ and $h \in L^{p, \sigma}$, then $g * h \in L^{p, \sigma}$.

(ii) Assume $1 \leq p, \sigma \leq \infty$ and $\sigma=p$ if $p=1$ or $\infty$. If $g \in L^{p^{\prime}, \sigma^{\prime}}$ and $h \in L^{p, \sigma}$, then $g * f \in$ $L^{\infty}$.

(iii) Assume $1<p<N$ and $1 \leq \sigma \leq \infty$. If $g \in L^{N p^{\prime} /\left(N+p^{\prime}\right), \sigma^{\prime}}$ and $h \in L^{p, \sigma}$, then $g * h \in$ $L^{N, 1}$.

Proof. (i) By interpolation, since the convolution with $g \in L^{1}$ is bounded on $L^{1}$ and on $L^{\infty}$.

(ii) By Hölder's inequality since $L^{p^{\prime}, \sigma^{\prime}}$ is the associated space of $L^{p, \sigma}$.

(ii) In general, if $1<p_{1}, p_{2}, p_{3}<\infty$ and $1 \leq \sigma_{1}, \sigma_{2}, \sigma_{3} \leq \infty$ and if $g \in L^{p_{1}, \sigma_{1}}$ and $h \in L^{p_{2}, \sigma_{2}}$, then $g * h \in L^{p_{3}, \sigma_{3}}$ if $1+1 / p_{3}=1 / p_{1}+1 / p_{2}$ and $1 / \sigma_{1}+1 / \sigma_{2}=1 / \sigma_{3}$. This is due to O'Neil [18] (as corrected by Yap [31]; see also Hunt [11]). To prove (ii), choose $p_{2}=p \in(1, N), \sigma_{2}=\sigma, p_{1}=N p^{\prime} /\left(N+p^{\prime}\right)>1, \sigma_{1}=\sigma^{\prime}, p_{3}=N, \sigma_{3}=1$.

The proof of Theorem 5.2 below will also involve the Sobolev spaces of negative infinite order $W^{-\infty, 1}$ and $W^{-\infty, \infty}$ (occasionally called $\mathcal{D}_{L^{1}}^{\prime}$ and $\mathcal{D}_{L^{\infty}}^{\prime}$, respectively). For convenience, we briefly recall their definition and a few relevant properties and refer to Schwartz [24, p. $199 \mathrm{ff}]$ for details.

For $1 \leq p \leq \infty$, define $W_{0}^{\infty, p}:=\cap_{m=0}^{\infty} W_{0}^{m, p}\left(=\cap_{m=0}^{\infty} W^{m, p}\right.$ if $\left.p<\infty\right)$, equipped with the $W^{m, p}$ norms, $m=0,1, \ldots$ and $W^{-\infty, p}:=\left(W_{0}^{\infty, p^{\prime}}\right)^{*}$ equipped with the seminorms $\sup _{u \in B}|T(u)|$ where $B$ is a bounded subset of $W_{0}^{\infty, p^{\prime}}$. It is readily checked that $W^{-\infty, p}=\cup_{m=0}^{\infty} W^{-m, p}$. In particular, every $T \in W^{-\infty, p}$ is a distribution and all the partial differentiations $\partial^{\gamma}\left(\gamma=:\left(\gamma_{1}, \ldots, \gamma_{N}\right)\right.$ a multi-index $)$ are continuous on $W^{-\infty, p}$. Also, $\mathcal{C}_{0}^{\infty}$ is dense in $W^{-\infty, p}$ if $p<\infty$.

Theorem 5.2. If $N>1$ and $\partial_{j} f \in L^{p_{j}, \sigma_{j}}$ with $1 \leq p_{j} \leq N$ and $\sigma_{j}=1$ if $p_{j}=1$ or $N$, the following properties hold. 
(i) $f \in W^{-\infty, \infty}$. Equivalently, $f \in \mathcal{S}^{\prime}$ (tempered distributions) and $f$ is a finite sum of derivatives of $L^{\infty}$ functions.

(ii) If $g \in W^{-\infty, 1}$, the convolution $g * f$ is defined in $W^{-\infty, \infty}$ and $\partial^{\alpha+\beta}(g * f)=\partial^{\alpha} g *$ $\partial^{\beta} f \in W^{-\infty, \infty}$ for every multi-indices $\alpha$ and $\beta$.

(iii) Set $p:=\min _{j} p_{j}$ and $\sigma:=\max _{p_{j}=p} \sigma_{j}$ and assume $g \in L^{1}$ if $p=N$, or $g \in L^{1} \cap$ $L^{N p^{\prime} /\left(N+p^{\prime}\right), \sigma^{\prime}}$ if $1<p<N$, or $g \in L^{1} \cap L^{N, 1}$ if $p=1$. Then, $g * f \in \mathcal{B U C}$ and there is a constant $c_{g * f} \in \mathbb{R}$ such that $\lim _{|x| \rightarrow \infty}(g * f)(x)=c_{g * f}$.

Proof. (i) If $\varphi \in \mathcal{C}_{0}^{\infty}$, the convolution $\varphi * f$ is defined in $L_{l o c}^{1}$ and $\partial_{j}(\varphi * f)=\varphi *$ $\partial_{j} f$. Next, $\varphi \in L^{1} \cap L^{p_{j}^{\prime}, \sigma_{j}^{\prime}}$ for $1 \leq j \leq N$, whence $\varphi * \partial_{j} f \in L^{p_{j}, \sigma_{j}} \cap L^{\infty} \subset L^{N, 1}$ by Lemma 5.1 (i) and (ii). Thus, $\nabla(\varphi * f) \in\left(L^{N, 1}\right)^{N}$ and so, by Theorem 1.1 (ii), $\varphi * f \in L^{\infty}$. It follows from [24, Theorem XXV, p. 201] that $f \in W^{-\infty, \infty}$ and that, equivalently, $f$ is a finite sum of derivatives of $L^{\infty}$ functions. In particular, $f \in \mathcal{S}^{\prime}$ since $L^{\infty} \subset \mathcal{S}^{\prime}$ and $\mathcal{S}^{\prime}$ is invariant under differentiation.

(ii) That the convolution $g * f$ is defined in $W^{-\infty, \infty}$ follows from (i) and from [24, Theorem XXVI, p. 203], where it is also shown that $(g, h) \in W^{-\infty, 1} \times W^{-\infty, \infty} \mapsto$ $g * h \in W^{-\infty, \infty}$ is continuous. In particular, $g \in W^{-\infty, 1} \mapsto g * \partial^{\beta} f \in W^{-\infty, \infty}$ is continuous. Hence, both $g \mapsto \partial^{\alpha+\beta}(g * f)$ and $g \mapsto \partial^{\alpha} g * \partial^{\beta} f$ are continuous linear mappings from $W^{-\infty, 1}$ to $W^{-\infty, \infty}$ and they coincide when $g=\varphi \in \mathcal{C}_{0}^{\infty}$. Since $\mathcal{C}_{0}^{\infty}$ is dense in $W^{-\infty, 1}$, they coincide when $g \in W^{-\infty, 1}$.

(iii) Since (obviously) $L^{1} \subset W^{-\infty, 1}$, it follows from (ii) that $g * f \in W^{-\infty, \infty}$ and that $\partial_{j}(g * f)=g * \partial_{j} f$. In particular, $\partial_{j}(g * f) \in L^{p_{j}, \sigma_{j}}$ by Lemma 5.1 (i). Thus, by Theorem 1.1 (ii) and Theorem 3.7, it suffices to show that $g * \partial_{j} f \in L^{N, 1}$ for every $j$ to prove that $g * f \in \mathcal{B U C}$ and that $\lim _{|x| \rightarrow \infty}(g * f)(x)=c_{g * f} \in \mathbb{R}$.

Assume $p=N$, so that $g \in L^{1}$ and $\partial_{j} f \in L^{N, 1}$ for every $j$. Hence, $g * \partial_{j} f \in L^{N, 1}$ by Lemma 5.1 (i).

Assume $1<p<N$, so that $g \in L^{1} \cap L^{N p^{\prime} /\left(N+p^{\prime}\right), \sigma^{\prime}}$. Then, $1<N p_{j}^{\prime} /\left(N+p_{j}^{\prime}\right) \leq N p^{\prime} /$ $\left(N+p^{\prime}\right)$ and $\sigma^{\prime} \leq \sigma_{j}^{\prime}$ when equality holds (use $p \leq p_{j}$ and $\sigma \geq \sigma_{j}$ when $p_{j}=p$ ). Therefore, $g \in L^{N p_{j}^{\prime} /\left(N+p_{j}^{\prime}\right), \sigma_{j}^{\prime}}$. In particular, $g * \partial_{j} f \in L^{N, 1}$ by Lemma 5.1 (iii) if $p_{j}<N$. If $p_{j}=N$, then $\partial_{j} f \in L^{N, 1}$ and so $g * \partial_{j} f \in L^{N, 1}$ by Lemma 5.1 (i).

Assume $p=1$, so that $g \in L^{1} \cap L^{N, 1}$. By Lemma 5.1 (i), $g * \partial_{j} f \in L^{N, 1}$ if $p_{j}=1$ or $N$ since, in the latter case, $\partial_{j} f \in L^{N, 1}$. If $1<p_{j}<N$, then $1<N p_{j}^{\prime} /\left(N+p_{j}^{\prime}\right)<N$, whence $g \in L^{N p_{j}^{\prime} /\left(N+p_{j}^{\prime}\right), \sigma_{j}^{\prime}}$ and $g * \partial_{j} f \in L^{N, 1}$ by Lemma 5.1 (iii).

Part (i) is false if $p_{j}>N$ for every $j$. Indeed, this condition holds if $f(x)=\log (1+$ $|x|)$. If $f$ is a finite sum of derivatives of $L^{\infty}$ functions, then $\varphi * f \in L^{\infty}$ for every $\varphi \in$ $\mathcal{C}_{0}^{\infty}$. However, if $\varphi \geq \chi_{B_{1}}$, then, $\varphi * f \geq \chi_{B_{1}} * f$, whence $\varphi * f \notin L^{\infty}$ since $\lim _{|x| \rightarrow \infty}\left(\chi_{B_{1}} *\right.$ $f)(x)=\infty$.

We illustrate Theorem 5.2 when $g$ is a Bessel kernel: 
Example 5.3. Assume $N>1$ and $\partial_{j} f \in L^{p_{j}, \sigma_{j}}$ with $1 \leq p_{j} \leq N$ and $\sigma_{j}=1$ if $p_{j}=1$ or $N$. By Theorem 5.2 (i), $f \in \mathcal{S}^{\prime}$ and so there is a unique solution $u_{\lambda} \in \mathcal{S}^{\prime}$ of $(I-$ $\Delta)^{\lambda / 2} u_{\lambda}=f$ for every $\lambda>0$, given by $u_{\lambda}=(I-\Delta)^{-\lambda / 2} f=G_{\lambda} * f$ where $G_{\lambda} \in L^{1}$ is the Bessel kernel of order $\lambda$.

Based on the behavior of $G_{\lambda}$ near the origin ([1, p. 416]) and near infinity (exponential decay), $G_{\lambda} \in L^{N p^{\prime} /\left(N+p^{\prime}\right), \sigma^{\prime}}$ with $p:=\min _{j} p_{j}>1$ and $\sigma:=\max _{p_{j}=p} \sigma_{j}$ if and only if $\lambda \geq N$ or $\lambda<N$ and $N p^{\prime} /\left(N+p^{\prime}\right)<N /(N-\lambda)$, that is, if and only if $\lambda>$ $N / p-1$. On the other hand, $G_{\lambda} \in L^{N, 1}$, needed if $p=1$, holds if and only if $\alpha>N-1$. Thus, the condition in Theorem 5.2 (iii) amounts to $\lambda>N / p-1$ irrespective of $p$ and, if so, $u_{\lambda} \in \mathcal{B U C}$ has a uniform finite constant limit at infinity.

If $\lambda=2 k$ with $k \in \mathbb{N}$ and if $\nabla f \in\left(L^{p}\right)^{N}$ with $1<p<N$ (i.e., $p_{j}=\sigma_{j}=p<N$ for every $j$ ), this also follows from the classical elliptic theory (see Remark 5.4 below), but there is no such theory if $p=1$ or if the $p_{j}$ are distinct, etc. Also, if $1 \leq p<N$, the above shows that $u_{2 k}=(I-\Delta)^{-k} f \in \mathcal{S}^{\prime}$ is bounded if $\nabla f \in\left(L^{p}\right)^{N}$ with $N /(2 k+1)<p<N$. This is optimal, except perhaps when $p=1$ and $N=2 k+1$ (notably, $N=3$ and $k=1$ ). Indeed, if $p \geq N>1$, a smooth function $u$ that coincides with $\log \log |x|$ for large $|x|$ is a counter-example. It is a little more demanding to produce a counter-example when $1 \leq p \leq N /(2 k+1)$; see the Appendix. In particular, it is noteworthy that if $N>2 k+1$, there is a window for the boundedness of $u_{2 k}$, which is generally false if $p$ is too small or too large.

Remark 5.4. If $\lambda=2 k$ with $k \in \mathbb{N}$ and if $\nabla f \in\left(L^{p}\right)^{N}$ with $1<p<N$, the condition $\lambda>-1+N / p$ in Example 5.3 is $p>N /(2 k+1)$ and, with $u:=u_{2 k}$ for simplicity, ( $I-$ $\Delta)^{k} \partial_{j} u=\partial_{j} f \in L^{p}$, so that $\partial_{j} u \in W^{2 k, p}$. Thus, $\partial_{j} u \in W^{1, p} \cap W^{2 k-1, p^{*}}$ and so $u-c_{u} \in$ $L^{p^{*}}$ for some $c_{u} \in \mathbb{R}$. Hence, $u-c_{u} \in W^{2 k, p^{*}} \subset \mathcal{B U C}$ (i.e., $\left.u \in \mathcal{B U C}\right)$ and $\lim _{|x| \rightarrow \infty}(u(x)-$ $\left.c_{u}\right)=0$ since $p>N /(2 k+1)$ is exactly $2 k p^{*}>N$.

By Lemma 5.1 (i), Theorem 1.1 (ii) and Theorem 3.7 are simultaneously applicable to $f$ and $g * f$ if $g \in L^{1}$. That the same thing is true of Theorem 4.7 is an immediate by-product of the following partial generalization of Young's theorem.

Lemma 5.5. Assume $1 \leq p, \sigma \leq \infty$ and $\sigma=p$ if $p=1$ or $\infty$. If $g \in L^{1}$ and $h \in$ $L_{u l o c}^{p, \sigma}$, then $g * h \in L_{u l o c}^{p, \sigma}$.

Proof. Since $L_{u l o c}^{\infty}=L^{\infty}$, there is nothing to prove when $p=\infty$. From now on, $1 \leq p<\infty$. We shall also assume $g \geq 0$ and $h \geq 0$ with no loss of generality. We proceed in three steps.

(a) Assume $p=1$ (hence $\sigma=1$ ), so that $h \in L_{u l o c}^{1}$ and the claim is that $g * h \in L_{u l o c}^{1}$. If $z \in \mathbb{R}^{N}$, then $\int_{B_{1}(z)}(g * h)(x) d x=\int_{\mathbb{R}^{N}} g(y) d y \int_{B_{1}(z)} h(x-y) d x$ by Tonelli's theorem. Now, $\quad \int_{B_{1}(z)} h(x-y) d x=\int_{B_{1}(z-y)} h \leq\|h\|_{1, u l o c}$ and so $\|g * h\|_{1, B_{1}(z)} \leq$ $\|g\|_{1}\|h\|_{1, u l o c}<\infty$. Since $z$ is arbitrary, this shows that $g * h \in L_{u l o c}^{1}$, as claimed. 
(b) Assume $1<\sigma \leq \infty$ (hence $1<p<\infty$ ). We begin with the proof that $g * h \in$ $L^{p, \sigma}\left(B_{1}(z)\right)$ for every $z \in \mathbb{R}^{N}$.

The space $L^{p, \sigma}\left(B_{1}(z)\right)$ is the topological dual of $L^{p^{\prime}, \sigma^{\prime}}\left(B_{1}(z)\right)$ for every $z \in \mathbb{R}^{N}$. Thus, to show that $g * h \in L^{p, \sigma}\left(B_{1}(z)\right)$, it suffices to show that $u(g * h) \in L^{1}\left(B_{1}(z)\right)$ for every $u \in L^{p^{\prime}, \sigma^{\prime}}\left(B_{1}(z)\right)$ and that $u \in L^{p^{\prime}, \sigma^{\prime}}\left(B_{1}(z)\right) \mapsto \int_{B_{1}(z)} u(g * h) \in \mathbb{R}$ is (linear and) continuous. To show $u(g * h) \in L^{1}\left(B_{1}(z)\right)$, it is not restrictive to assume $u \geq 0$. If so, once again by Tonelli's theorem,

$$
\int_{B_{1}(z)} u(g * h)=\int_{\mathbb{R}^{N}} g(y) d y \int_{B_{1}(z)} u(x) h(x-y) d x .
$$

Since $h \in L_{u l o c}^{p, \sigma}$, it follows that $h \in L^{p, \sigma}\left(B_{1}(z-y)\right)$ for every $y \in \mathbb{R}^{N}$, so that $h(\cdot-y) \in$ $L^{p, \sigma}\left(B_{1}(z)\right)$. In addition, $\|h(\cdot-y)\|_{p, \sigma, B_{1}(z)}=\|h\|_{p, \sigma, B_{1}(z-y)} \leq\|h\|_{p, \sigma, u l o c}$. Thus, by Hölder's inequality, $\quad \int_{B_{1}(z)} u(x) h(x-y) d x \leq\|u\|_{p^{\prime}, \sigma^{\prime}, B_{1}(z)}\|h\|_{p, \sigma, B_{1}(z-y)} \leq$ $\|u\|_{p^{\prime}, \sigma^{\prime}, B_{1}(z)}\|h\|_{p, \sigma, u l o c}$ and then $\int_{B_{1}(z)} u(g * h) \leq\|u\|_{p^{\prime}, \sigma^{\prime}, B_{1}(z)}\|g\|_{1}\|h\|_{p, \sigma, u l o c}<\infty$ by (5.1).

This shows that $u(g * h) \in L^{1}\left(B_{1}(z)\right)$ when $u \geq 0$ and, hence, in general. Furthermore, since $u$ and $|u|$ have the same $L^{p^{\prime}, \sigma^{\prime}}\left(B_{1}(z)\right)$ norm, $\left|\int_{B_{1}(z)} u(g * h)\right| \leq$ $\int_{B_{1}(z)}|u|(g * h) \leq\|u\|_{p^{\prime}, \sigma^{\prime}, B_{1}(z)}\|g\|_{1}\|h\|_{p, \sigma, u l o c}$ for every $u \in L^{p^{\prime}, \sigma^{\prime}}\left(B_{1}(z)\right)$. Thus, $u \in$ $L^{p^{\prime}, \sigma^{\prime}}\left(B_{1}(z)\right) \mapsto \int_{B_{1}(z)} u(g * h) \in \mathbb{R}$ is continuous. Consequently, $g * h \in L^{p, \sigma}\left(B_{1}(z)\right)$ and the dual norm $\|g * h\|_{p, \sigma, B_{1}(z)}^{\prime}$ is majorized by $\|g\|_{1}\|h\|_{p, \sigma, u l o c}$.

The norm $\|\cdot\|_{p, \sigma, B_{1}(z)}^{\prime}$ is equivalent to the (quasi-)norm $\|\cdot\|_{p, \sigma, B_{1}(z)}([2, \mathrm{p} .221])$, with constants independent of $z$ (by translation invariance). As a result, $\| g *$ $h\left\|_{p, \sigma, B_{1}(z)} \leq C\right\| g\left\|_{1}\right\| h \|_{p, \sigma, u l o c}$ with $C>0$ independent of $z$. This completes the proof that $g * h \in L_{u l o c}^{p, \sigma}$.

(c) Assume $\sigma=1$. Since the case $p=1$ was settled in (a), we also assume $1<p<\infty$. By arguing as in (b), we obtain

$$
\int_{B_{1}(z)}|u|(g * h) \leq\|u\|_{p^{\prime}, \infty, B_{1}(z)}\|g\|_{1}\|h\|_{p, 1, u l o c},
$$

for every $u \in L^{p^{\prime}, \infty}\left(B_{1}(z)\right)$ and every $z \in \mathbb{R}^{N}$, but the end of the proof must be modified since $L^{p, 1}\left(B_{1}(z)\right)$ is not the dual of $L^{p^{\prime}, \infty}\left(B_{1}(z)\right)$.

For simplicity, set $v:=(g * h)_{\mid B_{1}(z)} \geq 0$, so that (5.2) reads $\int_{B_{1}(z)}|u v| \leq$ $\|u\|_{p^{\prime}, \infty, B_{1}(z)}\|g\|_{1}\|h\|_{p, 1, u l o c}$ for every $u \in L^{p^{\prime}, \infty}\left(B_{1}(z)\right)$. If $\tilde{u}$ is a function on $B_{1}(z)$ equimeasurable with $u$, that is, $\tilde{u}^{*}=u^{*}$ (decreasing rearrangements), then $\tilde{u} \in$ $L^{p^{\prime}, \infty}\left(B_{1}(z)\right)$ and $\|\tilde{u}\|_{p^{\prime}, \infty, B_{1}(z)}=\|u\|_{p^{\prime}, \infty, B_{1}(z)}$. Thus, from the above, $\int_{B_{1}(z)}|\tilde{u} v| \leq$ $\|u\|_{p^{\prime}, \infty, B_{1}(z)}\|g\|_{1}\|h\|_{p, 1, u l o c}$. On the other hand, since $B_{1}(z)$ has finite measure, $\tilde{u}$ can be found such that $\int_{B_{1}(z)}|\tilde{u} v|=\int_{0}^{\infty} u^{*}(t) v^{*}(t) d t([2$, Theorem 2.6, p. 49]). 
Altogether, this yields

$$
\int_{0}^{\infty} u^{*}(t) v^{*}(t) d t \leq\|u\|_{p^{\prime}, \infty, B_{1}(z)}\|g\|_{1}\|h\|_{p, 1, u l o c}
$$

for every $u \in L^{p^{\prime}, \infty}\left(B_{1}(z)\right)$.

Choose $u(x):=|x-z|_{\mid B_{1}(z)}^{-N / p^{\prime}}$, so that $u^{*}(t)=\left|B_{1}\right|^{1 / p^{\prime}} t^{-1 / p^{\prime}}$ if $t<\left|B_{1}\right|$ and $u^{*}(t)=0$ if $t \geq\left|B_{1}\right|$. In particular, $\|u\|_{p^{\prime}, \infty, B_{1}(z)}=\left|B_{1}\right|^{1 / p^{\prime}}$. Likewise, $v^{*}(t)=0$ if $t \geq\left|B_{1}\right|$. Hence, $\int_{0}^{\infty} u^{*}(t) v^{*}(t) d t=\left|B_{1}\right|^{1 / p^{\prime}} \int_{0}^{\infty} t^{-1 / p^{\prime}} v^{*}(t) d t$ and so, by substitution into (5.3), $\int_{0}^{\infty} t^{-1 / p^{\prime}} v^{*}(t) d t \leq\|g\|_{1}\|h\|_{p, 1, u l o c}$, i.e., $\|v\|_{p, 1, B_{1}(z)} \leq\|g\|_{1}\|h\|_{p, 1, u l o c}$. Since $v:=(g *$ $h)_{\mid B_{1}(z)}$ and $z$ is arbitrary, it follows that $g * h \in L_{u l o c}^{p, 1}$.

Irrespective of $(p, \sigma)$ and of $q>1$, the convolution $g * h$ cannot be defined for all $h \in L_{\text {uloc }}^{p, \sigma}$ and all $g \in L^{q}$. To see this, choose $h=1 \in L_{u l o c}^{p, \sigma}$ and $g \in L^{q}$ such that $g_{ \pm} \in L^{q} \backslash L^{1}$. Then, $g_{ \pm} * 1=\int_{\mathbb{R}^{N}} g_{ \pm}=\infty$ and $g * 1$ does not make sense.

Theorem 5.6. If $g \in L^{1}$, every statement in Theorem 4.7 (Corollary 4.8) which is true for $f$ is true for $g * f$.

Proof. By Theorem 5.2 (ii), $\nabla^{i}(g * f)=g * \nabla^{i} f$ for every $i \geq 1$. Thus, by Lemma 5.1 (i) and Lemma 5.5, every assumption satisfied by $f$ in Theorem 4.7 is satisfied by $g * f$. Likewise, $\partial^{\gamma}(g * f)=g * \partial^{\gamma} f$ for every multi-index $\gamma$. Thus, by Young's theorem, every assumption satisfied by $f$ in Corollary 4.8 is satisfied by $g * f$.

The converse of Theorem 5.6 is false. In fact, there are so many ways for Theorem 4.7 or Corollary 4.8 to be applicable to $g * f$ but not necessarily to $f$ that a full classification is out of reach. For that reason, we only spell out a case when integrability conditions are placed only on the partial derivatives of $g \in L^{1}$ and, for simplicity, we confine attention to the Lebesgue space setting. For a more general statement, see Remark 5.8.

Theorem 5.7. Suppose that $\partial_{j} f \in L^{p_{j}}$ with $1 \leq p_{j}<N$ and set $p_{\min }:=\min _{j} p_{j}$ and $p_{\max }:=\max _{j} p_{j}$. Let $k \in[2, N+1]$ denote the smallest integer such that $p_{\min }>$ $N / k$ and suppose also that $g \in L^{1}$ and that $\partial^{\alpha} g \in L^{q_{\alpha}}$ with $1 \leq q_{\alpha} \leq p_{\max }^{\prime}$ when $1 \leq$ $|\alpha|_{1} \leq k-1$.

Then, $g * f \in \mathcal{B U C}$ and Theorem 3.1 (ii) is applicable to $g * f$. If also $p_{\max }<p^{\sharp}$ (see (4.4)), there is a constant $c_{g * f} \in \mathbb{R}$ such that $\lim _{|x| \rightarrow \infty}(g * f)(x)=c_{g * f}$.

Proof. The proof consists in showing that Corollary 4.8 is applicable to $g * f$. We shall use the fact that, by Theorem 5.2 (ii), $\partial^{\gamma}(g * f)$ with $|\gamma|_{1} \geq 1$ has the form $\partial^{\alpha} g * \partial_{j} f$ with $|\alpha|_{1}=|\gamma|_{1}-1$ and $1 \leq j \leq N$. 
First, $\partial_{j}(g * f)=g * \partial_{j} f \in L^{p_{j}}$ and condition (i) of Corollary 4.8 for $g * f$ is $\partial^{\gamma}(g *$ $f) \in L^{p_{\gamma}}$ for some $1 \leq p_{\gamma} \leq \infty$ if $1 \leq|\gamma|_{1} \leq k-1$. From the above, this is equivalent to $\partial^{\alpha} g * \partial_{j} f \in L^{p_{\alpha, j}}$ for some $1 \leq p_{\alpha, j} \leq \infty$ when $1 \leq j \leq N$ and $0 \leq|\alpha|_{1} \leq k-2$. This holds when $\alpha=0$; in particular, when $k=2$. If $k \geq 3$, it follows from Young's theorem that $p_{\alpha, j}$ exists if $\partial^{\alpha} g \in L^{q_{\alpha}}$ with $1 / q_{\alpha}+1 / p_{j} \geq 1$. This holds for every $1 \leq|\alpha|_{1} \leq k-2$ and every $1 \leq j \leq N$ if and only if $\max _{1 \leq|\alpha|_{1} \leq k-2} q_{\alpha} \leq p_{\max }^{\prime}$, (assumed)

Next, condition (ii) of Corollary 4.8 for $g * f$ is $\partial^{\gamma}(g * f) \in L^{p_{\gamma}}$ for some $N / k<$ $p_{\gamma} \leq \infty$ if $|\gamma|_{1}=k \leq N$, or $\partial^{\gamma}(g * f) \in L^{p_{\gamma}}$ for some $1 \leq p_{\gamma} \leq \infty$ if $k=N+1$ and $|\gamma|_{1}=$ $N+1$. Equivalently, $\partial^{\alpha} g * \partial_{j} f \in L^{p_{\alpha, j}}$ for some $N / k<p_{\alpha, j} \leq \infty$ if $k \leq N,|\alpha|_{1}=k-1$ and $1 \leq j \leq N$, or $\partial^{\alpha} g * \partial_{j} f \in L^{p_{\alpha, j}}$ for some $1 \leq p_{\alpha, j} \leq \infty$ if $k=N+1,|\alpha|_{1}=N$ and $1 \leq j \leq N$.

The first condition (when $k \leq N$ ) amounts to $1 \leq 1 / q_{\alpha}+1 / p_{j}<1+k / N$. This holds for every $|\alpha|_{1}=k-1$ and every $1 \leq j \leq N$ if and only if $\max _{1 \leq|\alpha|_{1}=k-1} q_{\alpha} \leq$ $p_{\max }^{\prime}$ (assumed) and $\min _{|\alpha|_{1}=k-1} q_{\alpha}>N p_{\min } /\left[(N+k) p_{\min }-N\right]$. This is not a restriction because $p_{\min }>N / k$ implies $N p_{\min } /\left[(N+k) p_{\min }-N\right]<1$. The second condition (when $k=N+1$ ) is equivalent to $\max _{1 \leq|\alpha|_{1}=N} q_{\alpha} \leq p_{\max }^{\prime}$ (assumed).

Remark 5.8. The proof shows that Theorem 5.7 remains true if $2 \leq k \leq N+1$ is any given integer, provided that $\min _{|\alpha|_{1}=k-1} q_{\alpha}>N p_{\min } /\left[(N+k) p_{\min }-N\right]$ if $k \leq N$ and $p_{\min } \leq N / k$. This may or may not be compatible with $q_{\alpha} \leq p_{\max }^{\prime}$.

As we already pointed out, Corollary 4.8 cannot be proved without the help of UL spaces. Therefore, it seems safe to say that no proof of Theorem 5.7 can be given based only on the properties of convolution and existing embedding theorems.

\section{A. Appendix}

Suppose $N>2 k+1$ with $k \in \mathbb{N}$ and let $1 \leq p \leq N /(2 k+1)$. In this appendix, we exhibit a function $f$ such that $\nabla f \in\left(L^{p}\right)^{N}$ and that the unique solution $u \in \mathcal{S}^{\prime}$ of $(I-\Delta)^{k} u=f$ is unbounded. This proves the optimality of the boundedness criterion in Example 5.3, except perhaps when $p=1$ and $N=2 k+1$.

If $h$ is a function on $\mathbb{R}^{N}$, we denote $h_{0}$ its restriction to $\mathbb{R}^{N} \backslash\{0\}$. On the other hand, functions on $\mathbb{R}^{N} \backslash\{0\}$ are also viewed as functions on $\mathbb{R}^{N}$ with no specified value at 0 . We need the following elementary lemma.

Lemma A.1. Let $h \in L_{\text {loc }}^{1}$ be smooth on $\mathbb{R}^{N} \backslash\{0\}$. If $\lim _{\varepsilon \rightarrow 0^{+}} \varepsilon^{N-1} h_{0}(\varepsilon \omega)=0$ uniformly for $\omega \in \mathbb{S}^{N-1}$ and if $\partial_{j} h_{0} \in L_{\text {loc }}^{1}$, then $\partial_{j} h=\partial_{j} h_{0}$ where $\partial_{j} h$ is the derivative of $h$ as a distribution on $\mathbb{R}^{N}$ and $\partial_{j} h_{0}$ is the classical derivative of the smooth function $h_{0}$.

Proof. If $\varphi \in \mathcal{C}_{0}^{\infty},\left\langle\partial_{j} h, \varphi\right\rangle=\lim _{\varepsilon \rightarrow 0^{+}} \int_{\mathbb{R}^{N} \backslash B_{\varepsilon}} \partial_{j} h_{0} \varphi-\int_{\mathbb{S}^{N-1}} \varepsilon^{N-1} h_{0}(\varepsilon \omega) \varphi(\varepsilon \omega) \omega_{j} d \omega$. From the assumptions $\lim _{\varepsilon \rightarrow 0^{+}} \varepsilon^{N-1} h_{0}(\varepsilon \omega)=0$ uniformly for $\omega \in \mathbb{S}^{N-1}$ and $\partial_{j} h_{0} \in$ $L_{\text {loc }}^{1}$, the right-hand side is $\int_{\mathbb{R}^{N}} \partial_{j} h_{0} \varphi$. 
Now, let $\psi \in \mathcal{C}_{0}^{\infty}\left(B_{1}\right)$ be nonnegative and equal to 1 on a neighborhood of 0 , so that the function $f(x):=\psi(x)|x|^{-2 k}\left(\log |x|^{-1}\right)^{-1}$ is smooth away from 0 and $f(x)=$ $|x|^{-2 k}\left(\log |x|^{-1}\right)^{-1}$ on a neighborhood of 0 . It is readily checked that $f \in L^{1}$ (recall $N>2 k+1)$ satisfies the hypotheses of Lemma A1, so that $\nabla f=\nabla f_{0}$. Furthermore, a straightforward verification reveals that $\nabla f_{0} \in\left(L^{p}\right)^{N}$ when $1 \leq p \leq N /(2 k+1)$ (this fails if $p=1$ and $N=2 k+1)$.

Let $u(x)=\int_{B_{1}} G_{2 k}(x-y) \psi(y)|y|^{-2 k}\left(\log |y|^{-1}\right)^{-1} d y$ be the unique solution of $(I-\Delta)^{k} u=f$ in $\mathcal{S}^{\prime}$. By local elliptic regularity, $u$ is continuous on $\mathbb{R}^{N} \backslash\{0\}$ (even $\mathcal{C}^{\infty}$ ) and, by Fatou's lemma, $u(0) \leq \liminf _{x \rightarrow 0} u(x)$. On the other hand, $u(0)=\infty$ since $G_{2 k}(-y) \psi(y)|y|^{-2 k}\left(\log |y|^{-1}\right)^{-1} \simeq|y|^{-N}\left(\log |y|^{-1}\right)^{-1}$ near $y=0$ and $\int_{B_{1}}|y|^{-N}\left(\log |y|^{-1}\right)^{-1} d y=\infty$. Thus, $\lim _{x \rightarrow 0} u(x)=\infty$ and so $u$ is unbounded.

\section{References}

1. Aronszajn, N. and Smith, K. T., Theory of Bessel potentials I, Ann. Inst. Fourier 11 (1961), 385-475.

2. Bennett, C. and Sharpley, R., Interpolation of Operators, Academic Press, Boston, 1988.

3. Bergh, J. and Löfström, J., Interpolation Spaces, Grund. Math. Wiss. 223, Springer, Berlin, 1976.

4. Cianchi, A. and Pick, L., Sobolev embeddings in BMO, VMO and $L_{\infty}$, Ark. Mat. 36 (1998), 317-340.

5. Deny, J. and Lions, J.-L., [Fr]Les espaces du type de Beppo Levi, Ann. Inst. Fourier 5 (1954), 305-370.

6. DeVore, R. A. and Sharpley, R. C., On the differentiability of functions in $\mathbb{R}^{n}$, Proc. Amer. Math. Soc. 91 (1984), 326-328.

7. DeVore, R. A. and Sherer, K., Interpolation of linear operators on Sobolev spaces, Ann. of Math. 109 (1979), 583-599.

8. Fefferman, C., Convergence on almost every line for functions with gradient in $L^{p}\left(\mathbb{R}^{n}\right)$, Ann. Inst. Fourier 24 (1974), 159-164.

9. Galdi, G. P., An Introduction to the Mathematical Theory of the Navier-Stokes Equations 1, Springer, New York, 1994.

10. Haškovec, J. and Schmeiser, C., A note on the anisotropic generalizations of the Sobolev and Morrey embedding theorems, Monatsh. Math. 158 (2009), 71-79.

11. Hunt, R. A., On $L(p, q)$ spaces, Enseign. Math. (2) 12 (1966), 249-275.

12. Kato, T., The Cauchy problem for quasi-linear symmetric hyperbolic systems, Arch. Ration. Mech. Anal. 58 (1975), 181-205.

13. Lieb, E. H. and Loss, M., Analysis, 2nd ed., Graduate Studies in Mathematics 14, AMS, Providence, 2001.

14. Lizorkin, P. I., Behavior at infinity of functions from Liouville classes. Riesz potentials of arbitrary order, Trudy Math. Inst. Steklov 150 (1979), 174-197 (in Russian).

15. Maz'JA, V. G., Sobolev Spaces, Springer, Berlin, 1985. 
16. Mizuta, Y., On the existence of limits along lines of Beppo Levi functions, Hiroshima Math. J. 16 (1986), 387-404.

17. Muckenhoupt, B. and Wheeden, R. L., On the dual of weighted $H^{1}$ of the halfspace, Studia Math. 63 (1978), 57-79.

18. O'NeIL, R., Convolution operators and $L(p, q)$ spaces, Duke Math. J. 30 (1963), 129 142.

19. Osserman, R., The isoperimetric inequality, Bull. Amer. Math. Soc. 84 (1978), 1182 1238.

20. Peetre, J., [Fr]Espaces d'interpolation et théorème de Soboleff, Ann. Inst. Fourier 16 (1966), 279-317.

21. RabieR, P. J., $L^{p}$ measure of growth and higher order Hardy-Sobolev-Morrey inequalities on $\mathbb{R}^{N}$, J. Math. Soc. Japan 69 (2017), 127-151. Erratum, J. Math. Soc. Japan 70 (2018), 877.

22. Rabier, P. J., Hölder continuity of Sobolev functions and Riesz potentials, Indiana Univ. Math. J. (to appear).

23. RÁkosník, J., Some remarks to anisotropic Sobolev spaces II, Beiträge Anal. 15 (1980), 127-140.

24. Schwartz, L., Théorie des distributions, Hermann, Paris, 1966.

25. Stein, E. M., Singular Integrals and Differentiability Properties of Functions, Princeton University Press, Princeton, 1970.

26. Stein, E. M., Editor's note: the differentiability of functions on $\mathbb{R}^{n}$, Ann. of Math. 113 (1981), 383-385.

27. Talenti, G., Inequalities in rearrangement invariant function spaces, in Nonlinear Analysis, Function Spaces and Applications 5, pp. 177-230, Prometheus, Prague, 1994.

28. TARTAR, L., Imbedding theorems of Sobolev spaces into Lorentz spaces, Boll. Unione Mat. Ital. (8) 1-B (1998), 479-500.

29. Turesson, B. O., Nonlinear Potential Theory and Weighted Sobolev Spaces, Lecture Notes in Math. 1736, Springer, Berlin, 2000.

30. Uspenskǐl, S. V., Imbedding theorems for weighted classes, Trudy Math. Inst. Steklov 60 (1961), 282-303 (in Russian).

31. YAP, L. Y. H., Some remarks on convolution operators and $L(p, q)$ spaces, Duke Math. J. 36 (1969), 647-658.

32. Ziemer, W. P., Weakly Differentiable Functions, Springer, New York, 1989.

Patrick J. Rabier

Department of Mathematics

University of Pittsburgh

Pittsburgh

PA 15260

U.S.A.

rabier@pitt.edu

Received June 9, 2017

in revised form December 28, 2017 